\title{
Design of Self-Tuning Regulator for Large-Scale Interconnected Hammerstein Systems
}

\author{
Mourad Elloumi and Samira Kamoun \\ National School of Engineers of Sfax (ENIS), Laboratory of Sciences and Techniques of Automatic Control \& Computer Engineering \\ (Lab-STA), University of Sfax, BP 1173, 3038 Sfax, Tunisia
}

Correspondence should be addressed to Mourad Elloumi; mourad.elloumi@yahoo.fr

Received 29 November 2015; Accepted 14 February 2016

Academic Editor: Ai-Guo Wu

Copyright (C) 2016 M. Elloumi and S. Kamoun. This is an open access article distributed under the Creative Commons Attribution License, which permits unrestricted use, distribution, and reproduction in any medium, provided the original work is properly cited.

\begin{abstract}
This paper deals with the self-tuning regulator for large-scale stochastic nonlinear systems, which are composed of several interconnected nonlinear monovariable subsystems. Each interconnected subsystem is described by discrete Hammerstein model with unknown and time-varying parameters. This self-tuning control is developed on the basis of the minimum variance approach and is combined by a recursive algorithm in the estimation step. The parametric estimation step is performed on the basis of the prediction error method and the least-squares techniques. Simulation results of the proposed self-tuning regulator for two interconnected nonlinear hydraulic systems show the reliability and effectiveness of the developed method.
\end{abstract}

\section{Introduction}

In the past few years, large-scale systems, which can be efficiently applied to many practical situations, such as power systems, transportation systems, industrial processes, and communication networks, have attracted much attention [125]. There exists extensive literature concerning control for large-scale systems. In this case, several results deal with some schemes for control of these systems on the basis of various approaches, such as minimum variance approach [7], generalized minimum variance strategy [8], decentralization $[1,2,12]$, sliding mode control technique [23], artificial neuronal networks, and fuzzy logical techniques [9-11].

Self-tuning regulators of stochastic systems with timevarying parameters are a problem of theoretical and practical importance. Since the synthesis of the first self-tuning regulator by Åström and Wittenmark in 1973, great numbers of theoretical and practical results are published in the literature in order to adjust automatically the regulator parameters online in response to change in the process and the environment. These results concerned more particularly the linear stochastic systems [7, 8, 26-28], the dual-rate sampled-data systems [29-31], and so forth, when various types of adaptive controllers are developed. In the field of adaptive control,
Kamoun presented optimal self-tuning regulators for largescale linear systems, which are described by discrete inputoutput mathematical models, based on minimum variance and generalized minimum variance strategies $[7,8]$; Zhang et al. developed a self-tuning control scheme based on multi-innovation stochastic gradient parameter estimation for discrete-time systems [28]; Ding et al. proposed an adaptive control algorithm for Hammerstein nonlinear dualrate systems [29]; and Ding and Chen developed least-squares and gradient based adaptive control algorithms for dual-rate linear systems $[30,31]$.

The minimum variance regulator was proposed by Åström and Wittenmark [26], which allows solving the regulation problem for a small size dynamic system described by an input-output mathematical model with known parameters. Afterwards, this approach has been extended by Kamoun for the control of large-scale linear systems, which are composed of several Single-Input Single-Output (SISO) interconnected subsystems and described by linear input-output mathematical models $[7,8]$. In the same way, Elloumi and Kamoun have considered the class of large-scale nonlinear systems, which are comprised of several SISO interconnected nonlinear subsystems, and developed an implicit self-tuning regulator for this class of dynamic systems [21]. 
Our objective is to develop a self-tuning regulator with explicit scheme which can be applied to the class of large-scale nonlinear systems with unknown time-varying parameters in order to resolve the regulation problem for this class of systems. We particularly focus on the dynamic large-scale nonlinear systems which are constituted by several SISO interconnected nonlinear systems and described by stochastic Hammerstein mathematical models with unknown timevarying parameters. The developed algorithm is combined by a recursive parametric estimation algorithm for determining the system parameters.

This paper is organized into four sections. The second section deals with the parametric estimation problem of the large-scale nonlinear systems operating in a stochastic environment. We focus here on the dynamic large-scale systems consisting of several interconnected nonlinear monovariable subsystems, which can be described by the class of Hammerstein mathematical models, with unknown and time-varying parameters. Section 3, which is the principal part of the paper, detailed the self-tuning control problem of the nonlinear interconnected subsystems based on the minimum variance approach. This control problem is formulated while being based on a control strategy minimizing a certain quadratic criterion. Finally, a simulation example is provided to illustrate the effectiveness and the efficiency of the proposed selftuning regulator scheme in Section 4. Conclusions are drawn in Section 5.

\section{Description and Parametric Estimation}

We consider a large-scale nonlinear system $S$ composed of $\mathcal{N}$ SISO interconnected nonlinear subsystems $S_{1}, \ldots, S_{\mathcal{N}}$. We assume that these nonlinear subsystems operate in a stochastic environment with unknown time-varying parameters. Thereby, the general structure of this considered system can be illustrated by Figure 1 .

The dynamic linear part of Hammerstein structure, as shown in Figure 1, is described by the following equation:

$$
\begin{aligned}
A_{i}\left(q^{-1}, k\right) y_{i}(k)= & q^{-d_{i}} B_{i}\left(q^{-1}, k\right) h_{i}^{u_{i}}(k) \\
& +\sum_{j=1, j \neq i}^{N} q^{-d_{i j}} B_{i j}\left(q^{-1}, k\right) h_{j}^{u_{j}}(k) \\
& +\sum_{j=1, j \neq i}^{N} q^{-t_{i j}} A_{i j}\left(q^{-1}, k\right) h_{j}^{y_{j}}(k) \\
& +C_{i}\left(q^{-1}\right) e_{i}(k),
\end{aligned}
$$

where $h_{i}^{u_{i}}(k)$ and $y_{i}(k)$ are, respectively, the input and the output of the dynamic linear part at the discrete-time $k, u_{i}(k)$, $u_{j}(k)$, and $y_{j}(k)$ represent the inputs of the nonlinear statics parts, $h_{j}^{u_{j}}(k)$ and $h_{j}^{y_{j}}(k)$ denote the inputs from the other interconnected dynamics systems $S_{j}, j=1, \ldots, N, j \neq i$, $\left\{e_{i}(k)\right\}$ is an independent random variable and is generated as Gaussian distribution with zero average and constant variance $\sigma_{i}^{2}, d_{i}$ is an intrinsic delay of the interconnected system $S_{i}, d_{i j}$ and $t_{i j}$ represent the delays of the interactions, which are related, respectively, to the inputs and the outputs of the other interconnected nonlinear systems $S_{j}, A_{i}\left(q^{-1}, k\right)$, $B_{i}\left(q^{-1}, k\right), A_{i j}\left(q^{-1}, k\right)$, and $B_{i j}\left(q^{-1}, k\right)$ are polynomials with unknown and time-varying parameters, and $C_{i}\left(q^{-1}\right)$ is a polynomial with unknown but constant parameters, which are defined by

$$
\begin{aligned}
A_{i}\left(q^{-1}, k\right) & =1+a_{i, 1}(k) q^{-1}+\cdots+a_{i, n_{A_{i}}}(k) q^{-n_{A_{i}},} \\
B_{i}\left(q^{-1}, k\right) & =b_{i, 1}(k) q^{-1}+\cdots+b_{i, n_{B_{i}}}(k) q^{-n_{B_{i}}}, \\
A_{i j}\left(q^{-1}, k\right) & =1+a_{i j, 1}(k) q^{-1}+\cdots+a_{i j, n_{A_{i j}}}(k) q^{-n_{A_{i j}}}, \\
B_{i j}\left(q^{-1}, k\right) & =b_{i j, 1}(k) q^{-1}+\cdots+b_{i j, n_{B_{i j}}}(k) q^{-n_{B_{i j}}}, \\
C_{i}\left(q^{-1}\right) & =1+c_{i, 1} q^{-1}+\cdots+c_{i, n_{C_{i}}} q^{-n_{C_{i}}},
\end{aligned}
$$

with $i, j=1, \ldots, N, j \neq i$, where $n_{A_{i}}, n_{B_{i}}, n_{A_{i j}}, n_{B_{i j}}$, and $n_{C_{i}}$ are the orders of the polynomials $A_{i}\left(q^{-1}, k\right), B_{i}\left(q^{-1}, k\right)$, $A_{i j}\left(q^{-1}, k\right), B_{i j}\left(q^{-1}, k\right)$, and $C_{i}\left(q^{-1}\right)$, respectively.

The nonlinear statics parts of the Hammerstein structure are represented by the following nonlinear functions:

$$
\begin{aligned}
& h_{i}^{u_{i}}(k)=f_{h_{i}^{u_{i}}}\left[u_{i}(k)\right], \\
& h_{j}^{u_{j}}(k)=f_{h_{j}^{u_{j}}}\left[u_{j}(k)\right], \\
& h_{j}^{y_{j}}(k)=f_{h_{j}^{y_{j}}}\left[y_{j}(k)\right],
\end{aligned}
$$

where $f_{h_{i}^{u_{i}}}[\cdot], f_{h_{j}^{u_{j}}}[\cdot]$, and $f_{h_{j}^{y_{j}}}[\cdot]$ are nonlinear functions.

In the literature, several presentations of these nonlinear functions are used like the polynomial functions that are frequently used in the description of these nonlinear elements. In this case, we propose to present these nonlinear functions by the following polynomials:

$$
\begin{aligned}
& h_{j}^{u_{i}}(k)=\sum_{r_{1}=1}^{p_{1}} \alpha_{i, r_{1}} u_{i}^{r_{1}}(k)+\Delta h_{i}^{u_{i}}\left[u_{i}(k)\right], \\
& h_{j}^{u_{j}}(k)=\sum_{r_{2}=1}^{p_{2}} \beta_{j, r_{2}} u_{j}^{r_{2}}(k)+\Delta h_{j}^{u_{j}}\left[u_{j}(k)\right], \\
& h_{j}^{y_{j}}(k)=\sum_{r_{3}=1}^{p_{3}} \gamma_{j, r_{3}} y_{j}^{r_{3}}(k)+\Delta h_{j}^{y_{j}}\left[y_{j}(k)\right],
\end{aligned}
$$

where $\Delta h_{i}^{u_{i}}\left[u_{i}(k)\right], \Delta h_{j}^{u_{j}}\left[u_{j}(k)\right]$, and $\Delta h_{j}^{y_{j}}\left[y_{j}(k)\right]$ represent approximated errors that can be assumed as noise acting on the system output. Their variances values depend on the chosen value of the nonlinearity degree $p_{t}, t=1,2,3$.

In order to facilitate the formulation of the parametric estimation problem for large-scale nonlinear systems, we retain the following assumptions. 


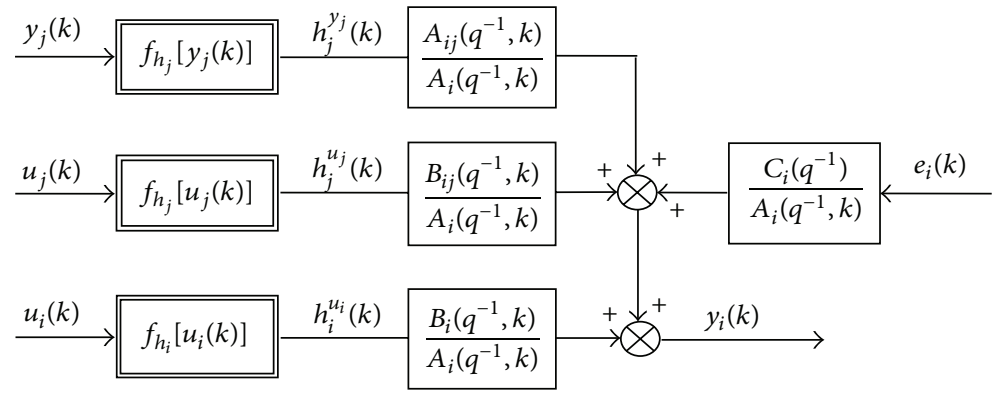

FIGURE 1: Structure of Hammerstein stochastic mathematical model.

Assumption 1. The choice of the nonlinearity degree is made in such a way that the approximated errors $\Delta h_{i}^{u_{i}}\left[u_{i}(k)\right]$, $\Delta h_{j}^{u_{j}}\left[u_{j}(k)\right]$, and $\Delta h_{j}^{y_{j}}\left[y_{j}(k)\right]$ can be neglected.

Assumption 2. The polynomials $A_{i}\left(q^{-1}, k\right), B_{i}\left(q^{-1}, k\right)$, $A_{i j}\left(q^{-1}, k\right), B_{i j}\left(q^{-1}, k\right)$, and $C_{i}\left(q^{-1}\right)$ of the mathematical model of the linear dynamic part have the same order $n_{i}$.

Taking into account these assumptions and using (1) and (4), we can express the system output as follows:

$$
\begin{aligned}
y_{i}(k)= & -\sum_{h=1}^{n_{i}} a_{i, h}(k) y_{i}(k-h) \\
& +\sum_{h=1}^{n_{i}} \sum_{r_{1}=1}^{p_{1}} b_{i, h}(k) \alpha_{i, r_{1}} u_{i}^{r_{1}}(k-h) \\
& +\sum_{j=1, j \neq i}^{N} \sum_{h=1}^{n_{i}} \sum_{r_{2}=1}^{p_{2}} b_{i j, h}(k) \beta_{j, r_{2}} u_{j}^{r_{2}}(k-h) \\
& +\sum_{j=1, j \neq i}^{N} \sum_{h=1}^{n_{i}} \sum_{r_{3}=1}^{p_{3}} a_{i j, h}(k) \gamma_{j, r_{3}} y_{j}^{r_{3}}(k-h) \\
& +\sum_{h=1}^{n_{i}} c_{i, h} e_{i}(k-h)+e_{i}(k),
\end{aligned}
$$

with $i, j=1, \ldots, N, j \neq i$.

The stochastic nonlinear system output $y_{i}(k)$, which is described by the Hammerstein mathematical model (5), can be rewritten in the following developed form:

$$
\begin{aligned}
y_{i}(k)= & -a_{i, 1}(k) y_{i}(k-1)-\cdots-a_{i, n_{i}}(k) y_{i}\left(k-n_{i}\right) \\
& +b_{i, 1}(k) \alpha_{i, 1} u_{i}(k-1)+b_{i, 2}(k) \alpha_{i, 1} u_{i}(k-2) \\
& +\cdots+b_{i, n_{i}}(k) \alpha_{i, 1} u_{i}\left(k-n_{i}\right)+\cdots \\
& +b_{i, 1}(k) \alpha_{i, p_{1}} u_{i}^{p_{1}}(k-1) \\
& +b_{i, 2}(k) \alpha_{i, p_{1}} u_{i}^{p_{1}}(k-2)+\cdots \\
& +b_{i, n_{i}}(k) \alpha_{i, p_{1}} u_{i}^{p_{1}}\left(k-n_{i}\right)
\end{aligned}
$$

$$
\begin{aligned}
& +b_{i j, 1}(k) \beta_{j, 1} u_{j}(k-1)+\cdots \\
& +b_{i j, n_{i}}(k) \beta_{j, 1} u_{j}\left(k-n_{i}\right)+\cdots \\
& +b_{i j, 1}(k) \beta_{j, p_{2}} u_{j}^{p_{2}}(k-1)+\cdots \\
& +b_{i j, n_{i}}(k) \beta_{j, p_{2}} u_{j}^{p_{2}}\left(k-n_{i}\right) \\
& +a_{i j, 1}(k) \gamma_{j, 1} y_{j}(k-1)+\cdots \\
& +a_{i j, n_{i}}(k) \gamma_{j, 1} y_{j}\left(k-n_{i}\right)+\cdots \\
& +a_{i j, 1}(k) \gamma_{j, p_{3}} y_{j}^{p_{3}}(k-1)+\cdots \\
& +a_{i j, n_{i}}(k) \gamma_{j, p_{3}} y_{j}^{p_{3}}\left(k-n_{i}\right)+e_{i}(k) \\
& +c_{i, 1} e_{i}(k-1)+\cdots+c_{i, n_{i}} e_{i}\left(k-n_{i}\right) .
\end{aligned}
$$

We remark that model (6) contains some redundant parameters, such as the parameters $b_{i, h}(k), b_{i j, h}(k)$, and $a_{i j, h}(k), h=1, \ldots, n_{i}$, which are nonlinear over the parameters $\alpha_{i, r_{1}}, \beta_{j, r_{2}}$, and $\gamma_{j, r_{3}}, r_{t}=1, \ldots, p_{t}, t=1,2,3, i, j=1, \ldots, N$, $j \neq i$, respectively. In that event, the formulation of the parametric estimation scheme presents some implementation difficulties. To overcome these difficulties, we proposed one of the following two situations [22]:

(1) To have knowledge about one of the parameters $b_{i, h}(k), b_{i j, h}(k)$, and $\gamma_{j, r_{3}}, h=1, \ldots, n_{i}, r_{3}=1, \ldots, p_{3}$. For example, when the parameters $b_{i, 1}(k), b_{i j, 1}(k)$, and $\gamma_{j, 1}$ are well known at the discrete-time $k$, we can estimate the parameters $\alpha_{i, r_{1}}(k), \beta_{j, r_{2}}(k)$, and $a_{i j, h}(k), r_{t}=1, \ldots, p_{t}, t=1,2, h=1, \ldots, n_{i}$. Thereafter, we can easily determine the parameters $\widehat{b}_{i, 2}(k), \ldots, \widehat{b}_{i, n_{i}}(k), \widehat{b}_{i j, 2}(k), \ldots, \widehat{b}_{i j, n_{i}}(k)$, and $\widehat{\gamma}_{j, r_{3}}(k)$, $r_{3}=2, \ldots, p_{3}$, based on the knowledge of $\widehat{\alpha}_{i, r_{1}}(k)$, $\widehat{\beta}_{j, r_{2}}(k)$, and $\widehat{a}_{i j, h}(k)$.

(2) To have knowledge about one of the parameters $\alpha_{i, r_{1}}, \beta_{j, r_{2}}$, and $\gamma_{j, r_{3}}, r_{t}=1, \ldots, p_{t}, t=1,2,3$. For example, in the case where the parameters $\alpha_{i, 1}$, $\beta_{j, 1}$, and $\gamma_{j, 1}$ are well known at the discrete-time $k$, we can estimate the parameters $b_{i, h}(k), b_{i j, h}(k)$, and $a_{i j, h}(k), h=1, \ldots, n_{i}$. Afterwards, based on the knowledge of these estimated parameters, we can 
easily determine the parameters $\widehat{\alpha}_{i, 2}(k), \ldots, \widehat{\alpha}_{i, p_{1}}(k)$, $\widehat{\beta}_{j, 2}(k), \ldots, \widehat{\beta}_{j, p_{2}}(k)$, and $\widehat{\gamma}_{j, 2}(k), \ldots, \widehat{\gamma}_{j, p_{3}}(k)$.

To overcome this problem, we assume, in what follows, that $b_{i, 1}(k), b_{i j, 1}(k)$, and $\gamma_{j, 1}$ are constant and known, in such a way that $b_{i, 1}(k)=b_{i j, 1}(k)=\gamma_{j, 1}=1, \forall k$. In this case, the system output $y_{i}(k)$ can be expressed by

$$
\begin{aligned}
y_{i}(k)= & -a_{i, 1}(k) y_{i}(k-1)-\cdots-a_{i, n_{i}}(k) y_{i}\left(k-n_{i}\right) \\
& +\alpha_{i, 1} u_{i}(k-1)+b_{i, 2}(k) \alpha_{i, 1} u_{i}(k-2)+\cdots \\
& +b_{i, n_{i}}(k) \alpha_{i, 1} u_{i}\left(k-n_{i}\right)+\cdots+\alpha_{i, p_{1}} u_{i}^{p_{1}}(k-1) \\
& +b_{i, 2}(k) \alpha_{i, p_{1}} u_{i}^{p_{1}}(k-2)+\cdots \\
& +b_{i, n_{i}}(k) \alpha_{i, p_{1}} u_{i}^{p_{1}}\left(k-n_{i}\right)+\beta_{j, 1} u_{j}(k-1) \\
& +\cdots+b_{i j, n_{i}}(k) \beta_{j, 1} u_{j}\left(k-n_{i}\right)+\cdots \\
& +\beta_{j, p_{2}} u_{j}^{p_{2}}(k-1)+\cdots \\
& +b_{i j, n_{i}}(k) \beta_{j, p_{2}} u_{j}^{p_{2}}\left(k-n_{i}\right) \\
& +a_{i j, 1}(k) y_{j}(k-1)+\cdots \\
& +a_{i j, n_{i}}(k) y_{j}\left(k-n_{i}\right)+\cdots \\
& +a_{i j, 1}(k) \gamma_{j, p_{3}} y_{j}^{p_{3}}(k-1)+\cdots \\
& +a_{i j, n_{i}}(k) \gamma_{j, p_{3}} y_{j}^{p_{3}}\left(k-n_{i}\right)+e_{i}(k) \\
& +c_{i, 1} e_{i}(k-1)+\cdots+c_{i, n_{i}} e_{i}\left(k-n_{i}\right),
\end{aligned}
$$

or equivalently in the matrix form

$$
y_{i}(k)=\theta_{i}^{T}(k) \psi_{i}(k)+e_{i}(k),
$$

where $\theta_{i}(k)$ and $\psi_{i}(k)$ represent, respectively, the parameter and the observations vectors, which are defined by

$$
\begin{aligned}
& \theta_{i}^{T}(k)=\left[\begin{array}{llllll}
a_{i, 1}(k) & \cdots & a_{i, n_{i}}(k) & \alpha_{i, 1} & \ell_{i, 21}(k) & \cdots
\end{array}\right.
\end{aligned}
$$

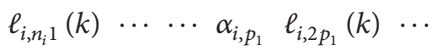

$$
\begin{aligned}
& \ell_{i, n_{i} p_{1}}(k) \beta_{j, 1} s_{i j, 21}(k) \cdots s_{i j, n_{i} 1}(k) \cdots \cdots \\
& \beta_{j, p_{2}} s_{i j, 2 p_{2}}(k) \cdots s_{i j, n_{i} p_{2}}(k) a_{i j, 1}(k)
\end{aligned}
$$

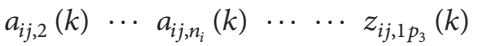

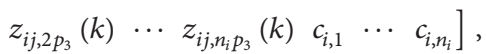

$$
\begin{aligned}
& \psi_{i}^{T}(k)=\left[\begin{array}{llll}
-y_{i}(k-1) & \cdots & -y_{i}\left(k-n_{i}\right) & u_{i}(k-1)
\end{array}\right. \\
& \begin{array}{lllllll}
u_{i}(k-2) & \cdots & u_{i}\left(k-n_{i}\right) & \cdots & \cdots & u_{i}^{p_{1}}(k-1)
\end{array} \\
& u_{i}^{p_{1}}(k-2) \cdots u_{i}^{p_{1}}\left(k-n_{i}\right) \quad u_{j}(k-1) \quad u_{j}(k-2) \cdots \\
& u_{j}\left(k-n_{i}\right) \cdots \cdots \cdots \\
& \begin{array}{lllllll}
u_{j}^{p_{2}}\left(k-n_{i}\right) & y_{j}(k-1) & y_{j}(k-2) & \cdots & y_{j}\left(k-n_{i}\right) & \cdots & \cdots
\end{array} \\
& y_{j}^{p_{3}}(k-1) \quad y_{j}^{p_{3}}(k-2) \cdots y_{j}^{p_{3}}\left(k-n_{i}\right) \\
& \left.e_{i}(k-1) e_{i}(k-2) \cdots e_{i}\left(k-n_{i}\right)\right] \text {, }
\end{aligned}
$$

with

$$
\begin{aligned}
& \ell_{i, 21}(k), \ldots, \ell_{i, n_{i} 1}(k)=b_{i, 2}(k) \alpha_{i, 1}, \ldots, b_{i, n_{i}}(k) \alpha_{i, 1}, \\
& \ell_{i, 2 p_{1}}(k), \ldots, \ell_{i, n_{i} p_{1}}(k)=b_{i, 2}(k) \alpha_{i, p_{1}}, \ldots, b_{i, n_{i}}(k) \alpha_{i, p_{1}}, \\
& s_{i j, 21}(k), \ldots, s_{i j, n_{i} 1}(k)=b_{i j, 2}(k) \beta_{j, 1}, \ldots, b_{i j, n_{i}}(k) \beta_{j, 1}, \\
& s_{i j, 2 p_{2}}(k), \ldots, s_{i j, n_{i} p_{2}}(k) \\
& \quad=b_{i j, 2}(k) \beta_{j, p_{2}}, \ldots, b_{i j, n_{i}}(k) \beta_{j, p_{2}}, \\
& z_{i j, 12}(k), \ldots, z_{i j, n_{i} 2}(k)=a_{i j, 1}(k) \gamma_{j, 2}, \ldots, a_{i j, n_{i}}(k) \gamma_{j, 2}, \\
& z_{i j, 1 p_{3}}(k), \ldots, z_{i j, n_{i} p_{3}}(k) \\
& =a_{i j, 1}(k) \gamma_{j, p_{3}}, \ldots, a_{i j, n_{i}}(k) \gamma_{j, p_{3}} .
\end{aligned}
$$

In the identification area of nonlinear systems, a large amount of research exploring different approaches has been published in the literature [22, 32-34]. Ding et al. developed an auxiliary model-based least-squares identification algorithm for nonlinear Hammerstein systems [32]. Ding and Chen presented an iterative and a recursive least-squares identification method for Hammerstein nonlinear ARMAX systems [33]. Recently, Ding et al. studied the parameter estimation problem of a class of nonlinear systems and proposed a RLS algorithm based on the model decomposition [34]. For the class of large-scale nonlinear systems, Elloumi and Kamoun developed an iterative estimator for identification of Hammerstein large-scale systems, based on prediction error method and least-squares techniques [22]. In this case, the estimate of the parameter vector $\theta_{i}(k)$, as defined by (9), is ensured starting from the following recursive extended leastsquares (RELS) algorithm [22]:

$$
\begin{aligned}
& \widehat{\theta}_{i}(k)=\widehat{\theta}_{i}(k-1)+P_{i}(k) \widehat{\psi}_{i}(k) \varepsilon_{i}(k), \\
& P_{i}(k)=\frac{1}{\lambda_{i}(k)}\left[P_{i}(k-1)\right. \\
& \left.-\frac{P_{i}(k-1) \widehat{\psi}_{i}(k) \widehat{\psi}_{i}^{T}(k) P_{i}(k-1)}{\lambda_{i}(k)+\widehat{\psi}_{i}^{T}(k) P_{i}(k-1) \widehat{\psi}_{i}(k)}\right], \\
& \varepsilon_{i}(k)=y_{i}(k)-\widehat{\theta}_{i}^{T}(k-1) \widehat{\psi}_{i}(k),
\end{aligned}
$$

where $\lambda_{i}(k)$ is the forgetting factor $\left(0 \prec \lambda_{i}(k) \prec 1\right), \widehat{\psi}_{i}(k)$ is the vector of the approximated observations $\psi_{i}(k)$, and $\widehat{\theta}_{i}(k)$ is the estimated parameters vector, which are described by

$$
\begin{aligned}
& \widehat{\psi}_{i}^{T}(k)=\left[\begin{array}{llll}
-y_{i}(k-1) & \cdots & -y_{i}\left(k-n_{i}\right) & u_{i}(k-1)
\end{array}\right. \\
& \begin{array}{llllll}
u_{i}(k-2) & \cdots & u_{i}\left(k-n_{i}\right) & \cdots & \cdots & u_{i}^{p_{1}}(k-1)
\end{array} \\
& u_{i}^{p_{1}}(k-2) \cdots u_{i}^{p_{1}}\left(k-n_{i}\right) u_{j}(k-1) u_{j}(k-2) \cdots \\
& u_{j}\left(k-n_{i}\right) \cdots \cdots \cdots \\
& u_{j}^{p_{2}}\left(k-n_{i}\right) \quad y_{j}(k-1) \quad y_{j}(k-2) \cdots \\
& y_{j}\left(k-n_{i}\right) \cdots \cdots \cdots \\
& \left.\begin{array}{llllll}
y_{j}^{p_{3}}\left(k-n_{i}\right) & \varepsilon_{i}(k-1) & \varepsilon_{i}(k-2) & \cdots & \varepsilon_{i}\left(k-n_{i}\right)
\end{array}\right],
\end{aligned}
$$




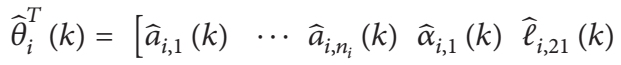

$$
\begin{aligned}
& \cdots \widehat{\ell}_{i, n_{i} 1}(k) \cdots \cdots \widehat{\alpha}_{i, p_{1}}(k) \hat{\ell}_{i, 2 p_{1}}(k) \cdots \\
& \widehat{\ell}_{i, n_{i} p_{1}}(k) \widehat{\beta}_{j, 1}(k) \widehat{s}_{i j, 21}(k) \cdots \widehat{s}_{i j, n_{i} 1}(k) \widehat{s}_{i j, 21}(k) \cdots \\
& \widehat{s}_{i j, n_{i} 1}(k) \cdots \cdots \widehat{\beta}_{j, p_{2}}(k) \widehat{s}_{i j, 2 p_{2}}(k) \cdots \\
& \widehat{s}_{i j, n_{i} p_{2}}(k) \widehat{a}_{i j, 1}(k) \hat{a}_{i j, 2}(k) \cdots \\
& \widehat{a}_{i j, n_{i}}(k) \cdots \cdots \widehat{z}_{i j, 1 p_{3}}(k) \widehat{z}_{i j, 2 p_{3}}(k) \cdots \\
& \left.\widehat{z}_{i j, n_{i} p_{3}}(k) \widehat{c}_{i, 1}(k) \cdots \cdots \widehat{c}_{i, n_{i}}(k)\right] \text {. }
\end{aligned}
$$

The practical implementation of the recursive extended least-squares (RELS) algorithm requires its initialization. Thus, we must give the values of the initial conditions of the estimated parameters vector $\theta_{i}(0)$ and the adaptation gain matrix $P_{i}(0)$. The convergence analysis of the RELS algorithm, which permits estimating the parameters of this class of systems, was proven by Elloumi and Kamoun [22].

\section{Minimum Variance Self-Tuning Regulation}

In this part, we propose to formulate the regulation problem of interconnected nonlinear systems, which can be described by Hammerstein stochastic mathematical model (1). In this case, we present an explicit self-tuning algorithm for the control of the subsystem $S_{i}$. This algorithm is obtained by combining a least-squares estimator of the explicit model. The purpose of the developed controller is to regulate the state of the overall system and to maintain stability in spite of the presence of nonlinear interconnections, environment variations, and ignorance and time variations of the parameters.

To develop an optimal self-tuning regulator for this class of large-scale systems, we retain these assumptions.

Assumption 1. The delays $d_{i}, d_{i j}$, and $t_{i j}$ are well known. In addition to that, we suppose also that $d_{i} \prec d_{i j}$ and $d_{i} \prec t_{i j}$ to facilitate the implementation of the control law $u_{i}(k)$.

Assumption 2. The parameters $b_{i, 1}(k), b_{i j, 1}(k)$, and $\gamma_{j, 1}$ are well known at each discrete-time $k$, in such a way that $b_{i, 1}(k)=$ $b_{i j, 1}(k)=\gamma_{j, 1}=1, \forall k$.

Assumption 3. The sequences $\left\{e_{i}(k)\right\}$ and $\left\{u_{i}(k), y_{i}(k), u_{j}(k)\right.$, $\left.y_{j}(k)\right\}$ are independent.

Let us consider a stochastic large-scale system, which consists of $\mathcal{N}$ SISO interconnected nonlinear subsystems $S_{1}, \ldots, S_{\mathcal{N}}$, with unknown time-varying parameters. This considered system can be described by the proposed Hammerstein model (1). Based on the cited assumptions, we can write the system output in the following form:

$$
\begin{aligned}
y_{i}(k)= & \frac{q^{-d_{i}} B_{i, 1}\left(q^{-1}, k\right)}{A_{i}\left(q^{-1}, k\right)} u_{i}(k) \\
& +\frac{q^{-d_{i}} B_{i, 2}\left(q^{-1}, k\right)}{A_{i}\left(q^{-1}, k\right)} u_{i}^{2}(k)+\cdots
\end{aligned}
$$

$$
\begin{aligned}
& +\frac{q^{-d_{i}} B_{i, p_{1}}\left(q^{-1}, k\right)}{A_{i}\left(q^{-1}, k\right)} u_{i}^{p_{1}}(k) \\
& +\frac{C_{i}\left(q^{-1}\right)}{A_{i}\left(q^{-1}, k\right)} e_{i}(k) \\
& +\frac{\sum_{j=1, j \neq i}^{N} \sum_{r_{2}=1}^{p_{2}} q^{-d_{i j}} B_{i j}\left(q^{-1}, k\right) \beta_{j, r_{2}}}{A_{i}\left(q^{-1}, k\right)} u_{j}^{r_{2}}(k) \\
& +\frac{\sum_{j=1, j \neq i}^{N} \sum_{r_{3}=1}^{p_{3}} q^{-t_{i j}} A_{i j}\left(q^{-1}, k\right) \gamma_{j, r_{3}}}{A_{i}\left(q^{-1}, k\right)} y_{j}^{r_{3}}(k),
\end{aligned}
$$

with $B_{i, r_{1}}\left(q^{-1}, k\right)=\alpha_{i, r_{1}} B_{i}\left(q^{-1}, k\right), r_{1}=1, \ldots, p_{1}, r_{2}=$ $1, \ldots, p_{2}$, and $r_{3}=1, \ldots, p_{3}$.

Or, equivalently, at the discrete-time $k+d_{i}+1$,

$$
\begin{aligned}
y_{i}\left(k+d_{i}+1\right)= & \frac{q B_{i, 1}\left(q^{-1}, k\right)}{A_{i}\left(q^{-1}, k\right)} u_{i}(k)+\frac{q B_{i, 2}\left(q^{-1}, k\right)}{A_{i}\left(q^{-1}, k\right)} \\
& \cdot u_{i}^{2}(k)+\cdots+\frac{q B_{i, p_{1}}\left(q^{-1}, k\right)}{A_{i}\left(q^{-1}, k\right)} \\
& \cdot u_{i}^{p_{1}}(k)+\frac{C_{i}\left(q^{-1}\right)}{A_{i}\left(q^{-1}, k\right)} e_{i}\left(k+d_{i}+1\right) \\
+ & \frac{\sum_{j=1, j \neq i}^{N} \sum_{r_{2}=1}^{p_{2}} B_{i j}\left(q^{-1}, k\right) \beta_{j, r_{2}}}{A_{i}\left(q^{-1}, k\right)} \\
& \cdot u_{j}^{r_{2}}\left(k-d_{i j}+d_{i}+1\right) \\
+ & \frac{\sum_{j=1, j \neq i}^{N} \sum_{r_{3}=1}^{p_{3}} A_{i j}\left(q^{-1}, k\right) \gamma_{j, r_{3}}}{A_{i}\left(q^{-1}, k\right)} \\
& \cdot y_{j}^{r_{3}}\left(k-t_{i j}+d_{i}+1\right) .
\end{aligned}
$$

The problem arising here consists in the development of a self-tuning regulator with explicit scheme that can be applied to the considered stochastic system, which is described by the discrete model (15). The formulation of this control problem can be ensured starting from the minimization of the following quadratic criterion:

$$
\begin{aligned}
J_{i}\left(k+d_{i}+1\right) \\
\quad=\hat{\mathrm{E}}\left[\left(y_{i}\left(k+d_{i}+1\right)-y_{i r}\left(k+d_{i}+1\right)\right)^{2}\right],
\end{aligned}
$$

where É represents the expected value and $y_{i r}\left(k+d_{i}+1\right)$ is the reference output of the considered system $S_{i}$.

By carrying out an Euclidian division of $C_{i}\left(q^{-1}\right) / A_{i}\left(q^{-1}\right.$, $k$ ) until the order $d_{i}$, we can write (15) as follows:

$$
\begin{gathered}
y_{i}\left(k+d_{i}+1\right)=\frac{q B_{i, 1}\left(q^{-1}, k\right)}{A_{i}\left(q^{-1}, k\right)} u_{i}(k)+\frac{q B_{i, 2}\left(q^{-1}, k\right)}{A_{i}\left(q^{-1}, k\right)} \\
\cdot u_{i}^{2}(k)+\cdots+\frac{q B_{i, p_{1}}\left(q^{-1}, k\right)}{A_{i}\left(q^{-1}, k\right)}
\end{gathered}
$$




$$
\begin{aligned}
& \cdot u_{i}^{p_{1}}(k)+\frac{G_{i}\left(q^{-1}, k\right)}{A_{i}\left(q^{-1}, k\right)} e_{i}(k) \\
+ & \frac{\sum_{j=1, j \neq i}^{N} \sum_{r_{2}=1}^{p_{2}} B_{i j}\left(q^{-1}, k\right) \beta_{j, r_{2}}}{A_{i}\left(q^{-1}, k\right)} \\
& \cdot u_{j}^{r_{2}}\left(k-d_{i j}+d_{i}+1\right) \\
+ & \frac{\sum_{j=1, j \neq i}^{N} \sum_{r_{3}=1}^{p_{3}} A_{i j}\left(q^{-1}, k\right) \gamma_{j, r_{3}}}{A_{i}\left(q^{-1}, k\right)} \\
& \cdot y_{j}^{r_{3}}\left(k-t_{i j}+d_{i}+1\right)+F_{i}\left(q^{-1}, k\right) \\
& \cdot e_{i}\left(k+d_{i}+1\right),
\end{aligned}
$$

where the polynomials $F_{i}\left(q^{-1}, k\right)$ and $G_{i}\left(q^{-1}, k\right)$ are defined by

$$
\begin{aligned}
F_{i}\left(q^{-1}, k\right)= & 1+f_{i, 1}(k) q^{-1}+\cdots+f_{i, d_{i}}(k) q^{-d_{i}} \\
G_{i}\left(q^{-1}, k\right)= & g_{i, 0}(k)+g_{i, 1}(k) q^{-1}+\cdots \\
& +g_{i, n_{i}-1}(k) q^{-n_{i}+1} .
\end{aligned}
$$

These two polynomials are solutions of the equation

$$
C_{i}\left(q^{-1}\right)=A_{i}\left(q^{-1}, k\right) F_{i}\left(q^{-1}, k\right)+q^{-d_{i}-1} G_{i}\left(q^{-1}, k\right) .
$$

Taking into account (14), the system output $y_{i}\left(k+d_{i}+1\right)$, as defined by (17), can be rewritten in the following form:

$$
\begin{aligned}
& y_{i}\left(k+d_{i}+1\right)=\frac{H_{i, 1}\left(q^{-1}, k\right)}{C_{i}\left(q^{-1}\right)} u_{i}(k)+\frac{H_{i, 2}\left(q^{-1}, k\right)}{C_{i}\left(q^{-1}\right)} \\
& \cdot u_{i}^{2}(k)+\cdots+\frac{H_{i, p_{1}}\left(q^{-1}, k\right)}{C_{i}\left(q^{-1}\right)} u_{i}^{p_{1}}(k)+\frac{G_{i}\left(q^{-1}, k\right)}{C_{i}\left(q^{-1}\right)} \\
& \cdot y_{i}(k) \\
& +\frac{\sum_{j=1, j \neq i}^{N} \sum_{r_{2}=1}^{p_{2}} B_{i j}\left(q^{-1}, k\right) F_{i}\left(q^{-1}, k\right) \beta_{j, r_{2}}}{C_{i}\left(q^{-1}\right)} \\
& \cdot u_{j}^{r_{2}}\left(k-d_{i j}+d_{i}+1\right) \\
& +\frac{\sum_{j=1, j \neq i}^{N} \sum_{r_{3}=1}^{p_{3}} A_{i j}\left(q^{-1}, k\right) F_{i}\left(q^{-1}, k\right) \gamma_{j, r_{3}}}{C_{i}\left(q^{-1}\right)} \\
& \quad y_{j}^{r_{3}}\left(k-t_{i j}+d_{i}+1\right)+F_{i}\left(q^{-1}, k\right) e_{i}\left(k+d_{i}+1\right),
\end{aligned}
$$

where the polynomial $H_{i, r_{1}}\left(q^{-1}, k\right)$ is described as follows:

$$
\begin{aligned}
H_{i, r_{1}}\left(q^{-1}, k\right)= & q B_{i, r_{1}}\left(q^{-1}, k\right) F_{i}\left(q^{-1}, k\right) \\
= & h_{i, r_{1} 1}(k)+h_{i, r_{1} 2}(k) q^{-1}+\cdots \\
& +h_{i, r_{1} n_{i}+d_{i}}(k) q^{-n_{i}-d_{i}+1},
\end{aligned}
$$

with $r_{1}=1, \ldots, p_{1}$ and $h_{i, r_{1} 1}(k)=b_{i, 1}(k) \alpha_{i, r_{1}}$.
We can show easily that the quadratic criteria $J_{i}\left(k+d_{i}+1\right)$, as given by (16), can be put in the following form:

$$
\begin{aligned}
& J_{i}\left(k+d_{i}+1\right)=\left[\frac{H_{i, 1}\left(q^{-1}, k\right)}{C_{i}\left(q^{-1}\right)} u_{i}(k)\right. \\
& +\frac{H_{i, 2}\left(q^{-1}, k\right)}{C_{i}\left(q^{-1}\right)} u_{i}^{2}(k)+\cdots+\frac{H_{i, p_{1}}\left(q^{-1}, k\right)}{C_{i}\left(q^{-1}\right)} u_{i}^{p_{1}}(k) \\
& +\frac{\sum_{j=1, j \neq i}^{N} \sum_{r_{2}=1}^{p_{2}} V_{i j, r_{2}}\left(q^{-1}, k\right)}{C_{i}\left(q^{-1}\right)} u_{j}^{r_{2}}\left(k-d_{i j}+d_{i}+1\right) \\
& +\frac{\sum_{j=1, j \neq i}^{N} \sum_{r_{3}=1}^{p_{3}} W_{i j, r_{3}}\left(q^{-1}, k\right)}{C_{i}\left(q^{-1}\right)} y_{j}^{r_{3}}\left(k-t_{i j}+d_{i}+1\right) \\
& \left.+\frac{G_{i}\left(q^{-1}, k\right)}{C_{i}\left(q^{-1}\right)} y_{i}(k)-y_{i r}\left(k+d_{i}+1\right)\right]^{2}+[1 \\
& \left.+f_{i, 1}^{2}(k)+f_{i, 2}^{2}(k)+\cdots+f_{i, d_{i}}^{2}(k)\right] \sigma_{i}^{2},
\end{aligned}
$$

where the polynomials $V_{i j, r_{2}}\left(q^{-1}, k\right)$ and $W_{i j, r_{3}}\left(q^{-1}, k\right)$ are defined as follows:

$$
\begin{aligned}
V_{i j, r_{2}}\left(q^{-1}, k\right)= & \beta_{j, r_{2}} B_{i j}\left(q^{-1}, k\right) F_{i}\left(q^{-1}, k\right) \\
= & v_{i j, r_{2} 1}(k) q^{-1}+\cdots \\
& +v_{i j, r_{2} n_{i}+d_{i}}(k) q^{-n_{i}-d_{i}}, \\
W_{i j, r_{3}}\left(q^{-1}, k\right)= & \gamma_{j, r_{3}} A_{i j}\left(q^{-1}, k\right) F_{i}\left(q^{-1}, k\right) \\
= & w_{i j, r_{3} 1}(k) q^{-1}+\cdots \\
& +w_{i j, r_{3} n_{i}+d_{i}}(k) q^{-n_{i}-d_{i}} .
\end{aligned}
$$

The optimal controller $u_{i}(k)$ making it possible to minimize the quadratic criterion, as given by (16), is obtained by solving the following equation:

$$
\begin{aligned}
& \frac{H_{i, 1}\left(q^{-1}, k\right)}{C_{i}\left(q^{-1}\right)} u_{i}(k)+\frac{H_{i, 2}\left(q^{-1}, k\right)}{C_{i}\left(q^{-1}\right)} u_{i}^{2}(k)+\cdots \\
& +\frac{H_{i, p_{1}}\left(q^{-1}, k\right)}{C_{i}\left(q^{-1}\right)} u_{i}^{p_{1}}(k) \\
& +\frac{\sum_{j=1, j \neq i}^{N} \sum_{r_{2}=1}^{p_{2}} V_{i j, r_{2}}\left(q^{-1}, k\right)}{C_{i}\left(q^{-1}\right)} u_{j}^{r_{2}}\left(k-d_{i j}+d_{i}+1\right)
\end{aligned}
$$

$$
\begin{aligned}
& +\frac{G_{i}\left(q^{-1}, k\right)}{C_{i}\left(q^{-1}\right)} y_{i}(k) \\
& +\frac{\sum_{j=1, j \neq i}^{N} \sum_{r_{3}=1}^{p_{3}} W_{i j, r_{3}}\left(q^{-1}, k\right)}{C_{i}\left(q^{-1}\right)} y_{j}^{r_{3}}\left(k-t_{i j}+d_{i}+1\right) \\
& -y_{i r}\left(k+d_{i}+1\right)=0 .
\end{aligned}
$$


Equation (25) can be written in the following polynomial form:

$$
\begin{aligned}
& M_{i, p_{1}}(k) u_{i}^{p_{1}}(k)+M_{i, p_{1}-1}(k) u_{i}^{p_{1}-1}(k)+\cdots \\
& \quad+M_{i, 2}(k) u_{i}^{2}(k)+M_{i, 1}(k) u_{i}(k)+M_{i, 0}(k)=0 .
\end{aligned}
$$

It should be noted that (25) admits real and/or complex solutions for each interconnected subsystem $S_{i}, 1 \leq i \leq N$. In this instance, we regard only the real solutions, in order to have a feasible controller $u_{i}(k)$. Moreover, if we get more than a real solution, the choice of the optimal control law $u_{i}(k)$, to minimize the effects of the noise acting on each output of the interconnected nonlinear system, can be conducted based on the following relation:

$$
\left|u_{i r}(k)-u_{i 0}\right|=\min ,
$$

where $u_{i r}(k)$ is the real solutions obtained starting from solving (25) and $u_{i 0}$ represents the optimal controller for the considered system, allowing the system output to follow the desired constant reference signal with a minimum variance output.

The implementation of the optimal control law $u_{i}(k)$ requires the knowledge of the different parameters involved in the polynomials $C_{i}\left(q^{-1}\right), \quad H_{i, r_{1}}\left(q^{-1}, k\right), \quad G_{i}\left(q^{-1}, k\right)$, $V_{i j, r_{2}}\left(q^{-1}, k\right)$, and $W_{i j, r_{3}}\left(q^{-1}, k\right), r_{1}=1, \ldots, p_{1}$. In this context, we need to add a parametric estimation step in the implementation.

In general, the minimum variance self-tuning regulator with explicit scheme can proceed by considering the following three steps.

Step 1. Estimate the different parameters involved in the Hammerstein mathematical model (1), based on the RELS algorithm.

Step 2. Determine the parameters of the polynomials $F_{i}\left(q^{-1}, k\right)$ and $G_{i}\left(q^{-1}, k\right)$ by resolution of the polynomial (19) and based on the estimated polynomials $\widehat{A}_{i}\left(q^{-1}, k\right)$ and $\widehat{C}_{i}\left(q^{-1}, k\right)$. We can also calculate the parameters of $H_{i, r_{1}}\left(q^{-1}, k\right), r_{1}=1, \ldots, p_{1}$, as given by (21), from the knowledge of the estimated parameters values $\widehat{\alpha}_{i, r_{1}}(k)$ and the values of the parameters involved in the polynomials $\widehat{B}_{i}\left(q^{-1}, k\right)$ and $F_{i}\left(q^{-1}, k\right)$. Similarly, from the knowledge of the estimated parameters values $\widehat{\beta}_{j, r_{2}}(k), r_{2}=1, \ldots, p_{2}$, and the values of the parameters involved in the polynomials $\widehat{B}_{i j}\left(q^{-1}, k\right)$ and $F_{i}\left(q^{-1}, k\right)$, we can calculate the parameters of the polynomial $V_{i j, r_{2}}\left(q^{-1}, k\right)$, as defined by (23). In addition to that, we can compute the parameters of the polynomial $W_{i j, r_{3}}\left(q^{-1}, k\right)$, as described by (24), from the knowledge of the estimated parameters values $\widehat{\gamma}_{j, r_{3}}(k), r_{3}=2, \ldots, p_{3}$, and the values of the parameters involved in the polynomials $\widehat{A}_{i j}\left(q^{-1}, k\right)$ and $F_{i}\left(q^{-1}, k\right)$.

Step 3. Compute the control law $u_{i}(k)$ starting from the resolution of polynomial (25) or (26).

It should be noted that the developed self-tuning regulator uses real-time estimation of the parameters of the

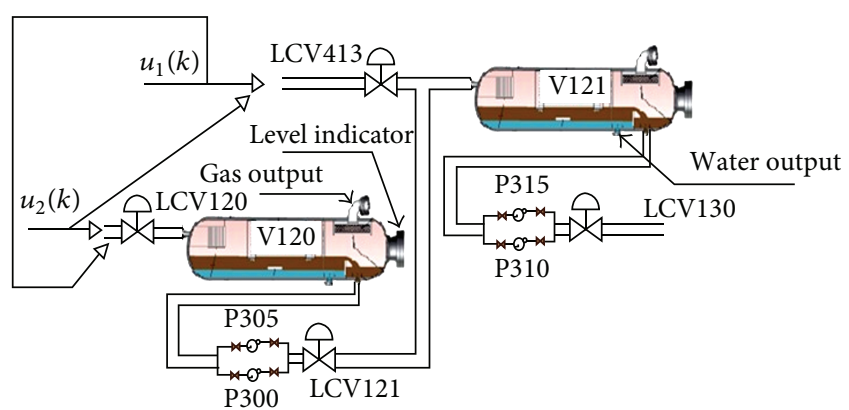

Figure 2: Oil circuit diagram of the considered interconnected hydraulic system.

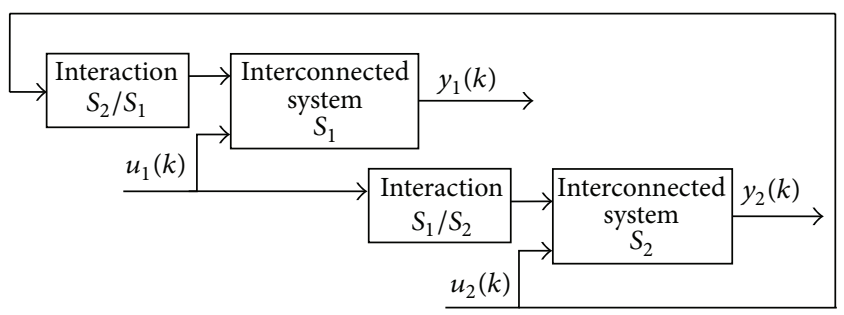

FIGURE 3: Interaction structure of the considered interconnected nonlinear subsystems $S_{1}$ and $S_{2}$.

interconnected nonlinear system $S_{i}, 1 \leq i \leq N$. In addition to that, the control law is obtained by solving (25) and based on relation (25), which must be made at each iteration step.

Let us note that the stability of the interconnected nonlinear system is ensured in closed loop if the controller $u_{i}(k)<\infty$ when $k$ tends to infinity. This occurs to show the conditions which allow obtaining a bounded control signal. These requirements can be achieved on the basis of an extended version of Kharitonov's method. This will be studied in a future research.

\section{Simulation Results}

The interconnected hydraulic system that we will deal with in this section is installed in petroleum society and depicted in Figure 2, in order to test the performance and the efficiency of the developed self-tuning regulator. This process is composed of two separators (V120 and V121), four motor-pumps shipments (P300, P305, P310, and P315), and four valves controls (LCV120, LCV121, LCV130, and LCV413). The docket of these separators is to deal with the fluid reservoir as it arrives from other separation stages, to segregate the three phases, water, oil, and gas, with stabilization of oil level in each one.

Figure 3 represents the interaction structure of the considered two interconnected subsystems $S_{1}$ and $S_{2}$.

This considered system $S_{i}, i=1,2$, can be described by the following mathematical model:

$$
\begin{aligned}
A_{i}\left(q^{-1}, k\right) y_{i}(k)= & q^{-1} B_{i}\left(q^{-1}, k\right) h_{i}^{u_{i}}(k) \\
& +\sum_{j=1, j \neq i}^{2} q^{-2} B_{i j}\left(q^{-1}, k\right) h_{j}^{u_{j}}(k) \\
& +C_{i}\left(q^{-1}\right) e_{i}(k),
\end{aligned}
$$


where the polynomials $A_{i}\left(q^{-1}, k\right), B_{i}\left(q^{-1}, k\right), B_{i j}\left(q^{-1}, k\right)$, and $C_{i}\left(q^{-1}\right)$ are defined by

$$
\begin{aligned}
A_{i}\left(q^{-1}, k\right) & =1+a_{i, 1}(k) q^{-1}+a_{i, 2}(k) q^{-2}, \\
B_{i}\left(q^{-1}, k\right) & =b_{i, 1}(k) q^{-1}+b_{i, 2}(k) q^{-2}, \\
B_{i j}\left(q^{-1}, k\right) & =b_{i j, 1}(k) q^{-1}+b_{i j, 2}(k) q^{-2}, \\
C_{i}\left(q^{-1}\right) & =1+c_{i, 1} q^{-1} .
\end{aligned}
$$

The nonlinear statics parts of the considered system are represented by the following polynomials:

$$
\begin{aligned}
& h_{i}^{u_{i}}(k)=\alpha_{i, 1} u_{i}(k)+\alpha_{i, 2} u_{i}^{2}(k), \\
& h_{j}^{u_{j}}(k)=\beta_{j, 1} u_{j}(k)+\beta_{j, 2} u_{j}^{2}(k) .
\end{aligned}
$$

We will apply in this simulation study the control method, developed in Section 3, to each interconnected nonlinear system $S_{i}$, which is described by the mathematical model (28). Thus, we can express the interconnected system output $y_{i}(k)$, $i=1,2$, by the following developed forms:

$$
\begin{aligned}
y_{1}(k)= & -a_{1,1}(k) y_{1}(k-1)-a_{1,2}(k) y_{1}(k-2) \\
& +\alpha_{1,1} u_{1}(k-2)+b_{1,2}(k) \alpha_{1,1} u_{1}(k-3) \\
& +\alpha_{1,2} u_{1}^{2}(k-2)+b_{1,2}(k) \alpha_{1,2} u_{1}^{2}(k-3) \\
& +\beta_{2,1} u_{2}(k-3)+b_{12,2}(k) \beta_{2,1} u_{2}(k-4) \\
& +\beta_{2,2} u_{2}^{2}(k-3)+b_{12,2}(k) \beta_{2,2} u_{2}^{2}(k-4) \\
& +e_{1}(k)+c_{1,1} e_{1}(k-1), \\
y_{2}(k)= & -a_{2,1}(k) y_{2}(k-1)-a_{2,2}(k) y_{2}(k-2) \\
& +\alpha_{2,1} u_{2}(k-2)+b_{2,2}(k) \alpha_{2,1} u_{2}(k-3) \\
& +\alpha_{2,2} u_{2}^{2}(k-2)+b_{2,2}(k) \alpha_{2,2} u_{2}^{2}(k-3) \\
& +\beta_{1,1} u_{1}(k-3)+b_{21,2}(k) \beta_{1,1} u_{1}(k-4) \\
& +\beta_{1,2} u_{1}^{2}(k-3)+b_{21,2}(k) \beta_{1,2} u_{1}^{2}(k-4) \\
& +e_{2}(k)+c_{2,1} e_{2}(k-1) .
\end{aligned}
$$

In this example of our numerical simulation, the noise sequences $\left\{e_{1}(k)\right\}$ and $\left\{e_{2}(k)\right\}$ are assumed to be independent and corresponding to a Gaussian distribution with zero mean and variances $\sigma_{1}^{2}=0.0845$ and $\sigma_{2}^{2}=0.0931$, respectively.

The output $y_{1}(k)$ of the interconnected nonlinear subsystem $S_{1}$ can be rewritten as follows:

$$
y_{1}(k)=\theta_{1}^{T}(k) \psi_{1}(k)+e_{1}(k),
$$

where the parameters vector $\theta_{1}(k)$ and the observations vector $\psi_{1}(k)$ are given as follows:

$$
\begin{aligned}
& \theta_{1}^{T}(k)=\left[\begin{array}{llllll}
a_{1,1}(k) & a_{1,2}(k) & \alpha_{1,1} & \ell_{1,21}(k) & \alpha_{1,2} & \ell_{1,22}(k) \\
\beta_{2,1} & s_{12,21}(k) & \beta_{2,2} & s_{12,22}(k) & c_{1,1}
\end{array}\right]
\end{aligned}
$$

$$
\begin{aligned}
& \psi_{1}^{T}(k)=\left[-y_{1}(k-1)-y_{1}(k-2) u_{1}(k-2) u_{1}(k-3)\right. \\
& u_{1}^{2}(k-2) u_{1}^{2}(k-3) u_{2}(k-3) u_{2}(k-4) \\
& \left.u_{2}^{2}(k-3) u_{2}^{2}(k-4) e_{1}(k-1)\right] \text {, }
\end{aligned}
$$

with $\ell_{1,21}(k)=b_{1,2}(k) \alpha_{1,1}, \ell_{1,22}(k)=b_{1,2}(k) \alpha_{1,2}, s_{12,21}(k)=$ $b_{12,2}(k) \beta_{2,1}$, and $s_{12,22}(k)=b_{12,2}(k) \beta_{2,2}$.

Similarly, the output $y_{2}(k)$ of the interconnected nonlinear subsystem $S_{2}$ can be rewritten in the following form:

$$
y_{2}(k)=\theta_{2}^{T}(k) \psi_{2}(k)+e_{2}(k),
$$

where the parameters vector $\theta_{2}(k)$ and the observations vector $\psi_{2}(k)$ are described by

$$
\begin{aligned}
& \theta_{2}^{T}(k)=\left[\begin{array}{llllll}
a_{2,1}(k) & a_{2,2}(k) & \alpha_{2,1} & \ell_{2,21}(k) & \alpha_{2,2} & \ell_{2,22}(k) \\
\beta_{1,1} & s_{21,21}(k) & \beta_{1,2} & s_{21,22}(k) & c_{2,1}
\end{array}\right], \\
& \psi_{2}^{T}(k)=\left[\begin{array}{lllll}
-y_{2}(k-1) & -y_{2}(k-2) & u_{2}(k-2) & u_{2}(k-3) \\
u_{2}^{2}(k-2) & u_{2}^{2}(k-3) & u_{1}(k-3) & u_{1}(k-4) \\
u_{1}^{2}(k-3) & u_{1}^{2}(k-4) & e_{2}(k-1)
\end{array}\right],
\end{aligned}
$$

with $\ell_{2,21}(k)=b_{2,2}(k) \alpha_{2,1}, \ell_{2,22}(k)=b_{2,2}(k) \alpha_{2,2}, s_{21,21}(k)=$ $b_{21,2}(k) \beta_{1,1}$, and $s_{21,22}(k)=b_{21,2}(k) \beta_{1,2}$.

In the first step of the control method, we propose to estimate the parameters involved in the parameter vectors (34) and (37) using the RELS algorithm (12). The second step is reserved to the resolution of the polynomial equation:

$$
C_{i}\left(q^{-1}\right)=A_{i}\left(q^{-1}, k\right) F_{i}\left(q^{-1}, k\right)+q^{-2} G_{i}\left(q^{-1}, k\right) .
$$

The resolution of (39) permits determining the parameters of the polynomials:

$$
\begin{aligned}
F_{i}\left(q^{-1}, k\right) & =1+f_{i, 1}(k) q^{-1}, \\
G_{i}\left(q^{-1}, k\right) & =g_{i, 0}(k)+g_{i, 1}(k) q^{-1},
\end{aligned}
$$

with

$$
\begin{aligned}
& f_{i, 1}(k)=\widehat{c}_{i, 1}(k)-\widehat{a}_{i, 1}(k), \\
& g_{i, 0}(k)=-\widehat{a}_{i, 1}(k) f_{i, 1}(k)-\widehat{a}_{i, 2}(k), \\
& g_{i, 1}(k)=-\widehat{a}_{i, 2}(k) f_{i, 1}(k) .
\end{aligned}
$$

As for the third step, it concerns resolving the following equation:

$$
M_{i, 2}(k) u_{i}^{2}(k)+M_{i, 1}(k) u_{i}(k)+M_{i, 0}(k)=0,
$$

where

$$
\begin{aligned}
M_{i, 2}(k)= & h_{i, 21}(k), \\
M_{i, 1}(k)= & h_{i, 11}(k), \\
M_{i, 0}(k)= & h_{i, 12}(k) u_{i}(k-1)+h_{i, 13}(k) u_{i}(k-2) \\
& +h_{i, 22}(k) u_{i}^{2}(k-1) \\
& +h_{i, 23}(k) u_{i}^{2}(k-2)+g_{i, 0}(k) y_{i}(k) \\
& +g_{i, 1}(k) y_{i}(k-1)+v_{i j, 11}(k) u_{j}(k-1)
\end{aligned}
$$




$$
\begin{aligned}
& +v_{i j, 12}(k) u_{j}(k-2) \\
& +v_{i j, 13}(k) u_{j}(k-3) \\
& +v_{i j, 21}(k) u_{j}^{2}(k-1) \\
& +v_{i j, 22}(k) u_{j}^{2}(k-2) \\
& +v_{i j, 23}(k) u_{j}^{2}(k-3)-y_{i r}(k+2) \\
& -\widehat{c}_{i, 1}(k) y_{i r}(k+1),
\end{aligned}
$$

with

$$
\begin{aligned}
& h_{i, 11}(k)=\widehat{\alpha}_{i, 1}(k), \\
& h_{i, 12}(k)=\widehat{\alpha}_{i, 1}(k)\left(f_{i, 1}(k)+\widehat{b}_{i, 2}(k)\right), \\
& h_{i, 13}(k)=\widehat{\alpha}_{i, 1}(k) \widehat{b}_{i, 2}(k) f_{i, 1}(k), \\
& h_{i, 21}(k)=\widehat{\alpha}_{i, 2}(k), \\
& h_{i, 22}(k)=\widehat{\alpha}_{i, 2}(k)\left(f_{i, 1}(k)+\widehat{b}_{i, 2}(k)\right), \\
& h_{i, 23}(k)=\widehat{\alpha}_{i, 2}(k) \widehat{b}_{i, 2}(k) f_{i, 1}(k), \\
& v_{i j, 11}(k)=\widehat{\beta}_{j, 1}(k), \\
& v_{i j, 12}(k)=\widehat{\beta}_{j, 1}(k)\left(f_{i, 1}(k)+\widehat{b}_{i j, 2}(k)\right), \\
& v_{i j, 13}(k)=f_{i, 1}(k) \widehat{b}_{i j, 2}(k) \widehat{\beta}_{j, 1}(k), \\
& v_{i j, 21}(k)=\widehat{\beta}_{j, 2}(k), \\
& v_{i j, 22}(k)=\widehat{\beta}_{j, 2}(k)\left(f_{i, 1}(k)+\widehat{b}_{i j, 2}(k)\right), \\
& v_{i j, 23}(k)=f_{i, 1}(k) \widehat{b}_{i j, 2}(k) \widehat{\beta}_{j, 2}(k),
\end{aligned}
$$

with $i, j=1,2, j \neq i$.

Based on discriminant $\Delta=M_{i, 1}^{2}(k)-4 M_{i, 2}(k) M_{i, 0}(k)$, the control law $u_{i}(k)$ is given, such that

(i) if $\Delta>0$, then $u_{i}(k)=\left(-M_{i, 1}(k) \pm\right.$ $\left.\sqrt{M_{i, 1}^{2}(k)-4 M_{i, 2}(k) M_{i, 0}(k)}\right) / 2 M_{i, 2}(k)$;

(ii) if $\Delta=0$, then $u_{i}(k)=-M_{i, 1}(k) / 2 M_{i, 2}(k)$;

(iii) if $\Delta$ is strictly negative, then the polynomial (42) does not admit solution. In this case, we propose that $u_{i}(k)=u_{i}(k-1)$.

It can be remarked that the boundedness of these obtained controllers depends on the parameter $M_{i, 2}(k)=$ $h_{i, 21}(k)$. However, if this parameter is equal to zero at discretetime $k_{0}$, then the control law $u_{i}\left(k_{0}\right)$ diverges. To overcome this problem, we must append a test on the estimated parameter value $\widehat{\alpha}_{i, 2}(k)$. For example, if $\widehat{\alpha}_{i, 2}\left(k_{0}\right)=0$, we suppose that $\widehat{\alpha}_{i, 2}\left(k_{0}\right)=0,1$ in order to complete the computation of the controller $u_{i}\left(k_{0}\right)$ involved in the iterative control algorithm.

The data for the practical implementation of the developed self-tuning regulator is given below:
TABLE 1: Statistical average values of the interconnected system.

\begin{tabular}{lccc}
$\bar{m}_{\varepsilon_{1}(k)}$ & $\sigma_{\varepsilon_{1}(k)}^{2}$ & $\bar{m}_{\varepsilon_{2}(k)}$ & $\sigma_{\varepsilon_{2}(k)}^{2}$ \\
0.0041 & 0.1125 & -0.0053 & 0.0885 \\
\hline $\bar{m}_{D_{1}(k)}$ & $\sigma_{D_{1}(k)}^{2}$ & $\bar{m}_{D_{2}(k)}$ & $\sigma_{D_{2}(k)}^{2}$ \\
0.2452 & 0.1367 & 0.2438 & 0.3147
\end{tabular}

(1) The parameters values intervening in the mathematical model (31) are chosen as follows: $a_{1,1}(k)=-0.88+$ $0.02 \sin (0.2 k), a_{1,2}(k)=0.45+0.03 \cos (0.2 k), b_{1,2}(k)=$ $0.32+0.03 \sin (0.2 k), c_{1,1}=0.25, b_{12,2}(k)=0.33+$ $0.04 \sin (0.2 k), \alpha_{1,1}=0.28, \alpha_{1,2}=0.22, \beta_{2,1}=0.33$, and $\beta_{2,2}=0.23$.

(2) The parameters values of the mathematical model (32) are selected as follows: $a_{2,1}(k)=-0.85+$ $0.02 \sin (0.2 k), a_{2,2}(k)=0.4+0.03 \cos (0.2 k), b_{2,2}(k)=$ $0.35+0.03 \sin (0.2 k), c_{2,1}=0.3, b_{21,2}(k)=0.48+$ $0.04 \sin (0.2 k), \alpha_{2,1}=0.33, \alpha_{2,2}=0.23, \beta_{1,1}=0.28$, and $\beta_{1,2}=0.22$.

(3) The initial conditions of the estimated parameters vector $\widehat{\theta}_{i}(0)$ and the adaptation gain matrix $P_{i}(0)$ are chosen in such a way that $\widehat{\theta}_{i}(0)=0$ and $P_{i}(0)=1000 I$, where $I$ is the identity matrix, $i=1,2$.

(4) The forgetting factors $\lambda_{1}(k)$ and $\lambda_{2}(k)$ are chosen in such a way that $\lambda_{1}(k)=0.995$ and $\lambda_{2}(k)=0.992$.

(5) The number of measurements is selected as $M=$ $1, \ldots, 600$.

(6) The two control laws $u_{1}(k)$ and $u_{2}(k)$ must minimize the following quadratic criteria:

$$
\begin{aligned}
& J_{i}(k+2)=\mathrm{E}\left[\left(y_{i}(k+2)-y_{i r}(k+2)\right)^{2}\right] \text {, } \\
& \text { with } y_{1 r}(k)=3.5 \text { and } y_{2 r}(k)=4 \text {. }
\end{aligned}
$$

Some results of this simulation example of the considered system $S_{i}, i=1,2$, are given. Thus, Figures 4 and 5 represent the evolution curves of the prediction error, the parametric distance, and their overall variances for each interconnected nonlinear subsystem.

Table 1 presents the statistical average values of the prediction errors $\varepsilon_{1}(k)$ and $\varepsilon_{2}(k)$, the parametric distances $D_{1}(k)$ and $D_{2}(k)$, and their overall variances $\sigma_{\varepsilon_{1}}^{2}(k), \sigma_{D_{1}}^{2}(k), \sigma_{\varepsilon_{2}}^{2}(k)$, and $\sigma_{D_{2}}^{2}(k)$, where the statistical average and the variance of the different sizes illustrated in Table 1 can be made starting from the calculation of the following expressions:

$$
\begin{aligned}
\bar{m}_{X} & =\frac{\sum_{k=401}^{600}[X]}{200}, \\
\sigma_{X}^{2} & =\frac{\sum_{k=401}^{600}\left[X-\bar{m}_{X}\right]^{2}}{200} .
\end{aligned}
$$

It can be remarked that the parametric distances (statistical average) have some quite low values. We can remark also that the shapes of prediction errors have some relatively low values too. Consequently, the evolution curves of the different 

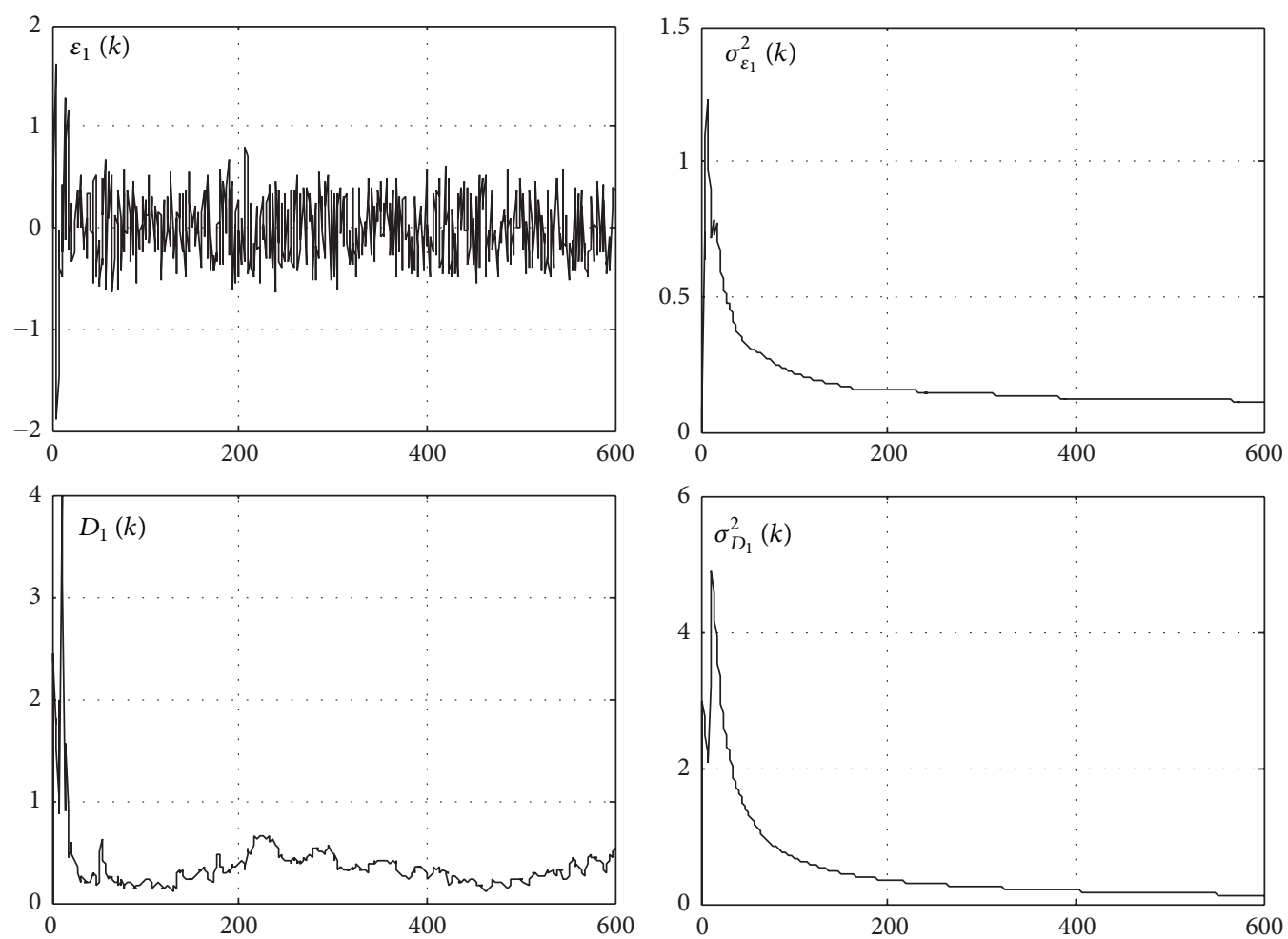

FIGURE 4: Evolution curves of the prediction error $\varepsilon_{1}(k)$, the parametric distance $D_{1}(k)$, and their overall variances $\sigma_{\varepsilon_{1}}^{2}(k)$ and $\sigma_{D_{1}}^{2}(k)$.
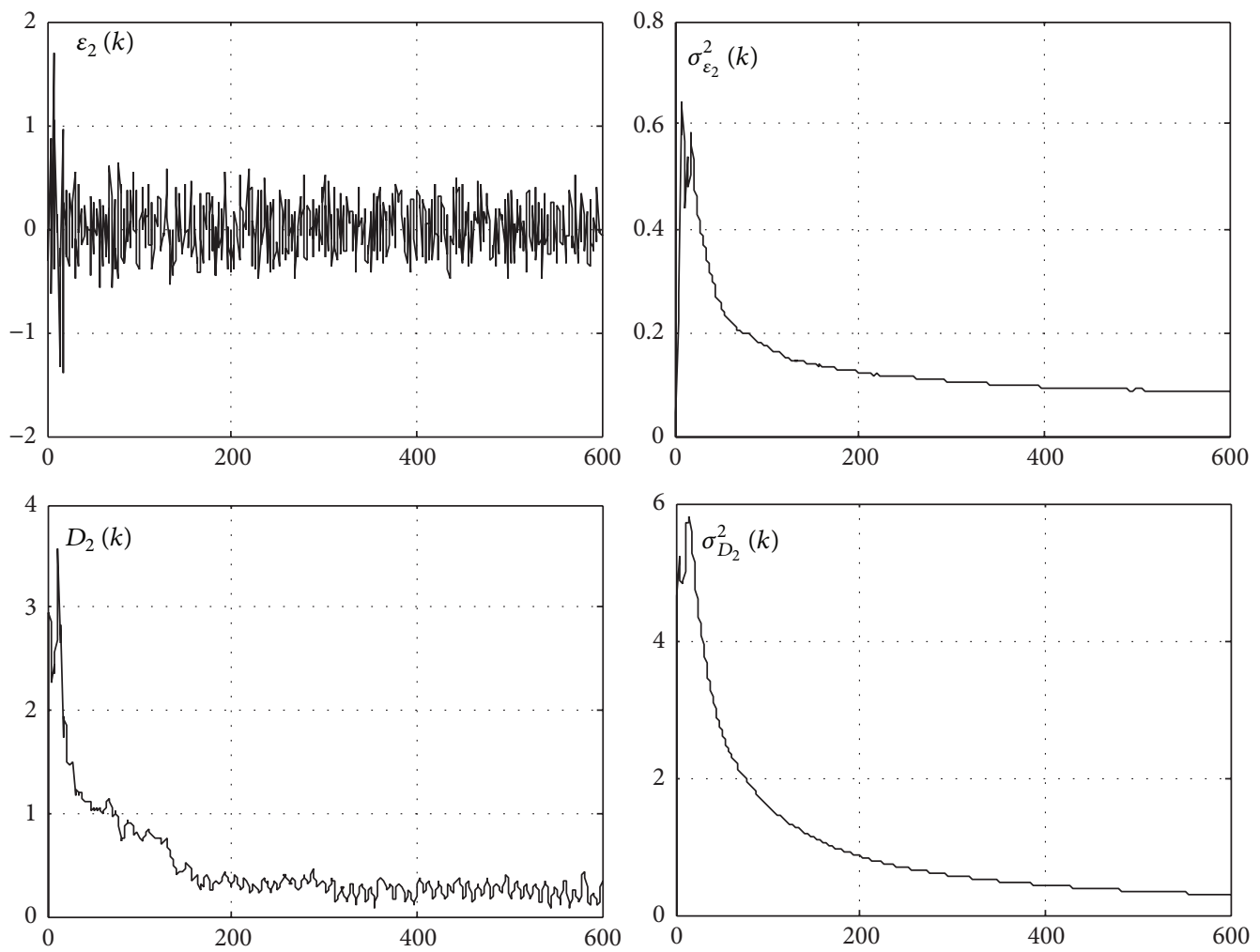

FIGURE 5: Evolution curves of the prediction error $\varepsilon_{2}(k)$, the parametric distance $D_{2}(k)$, and their overall variances $\sigma_{\varepsilon_{2}}^{2}(k)$ and $\sigma_{D_{2}}^{2}(k)$. 

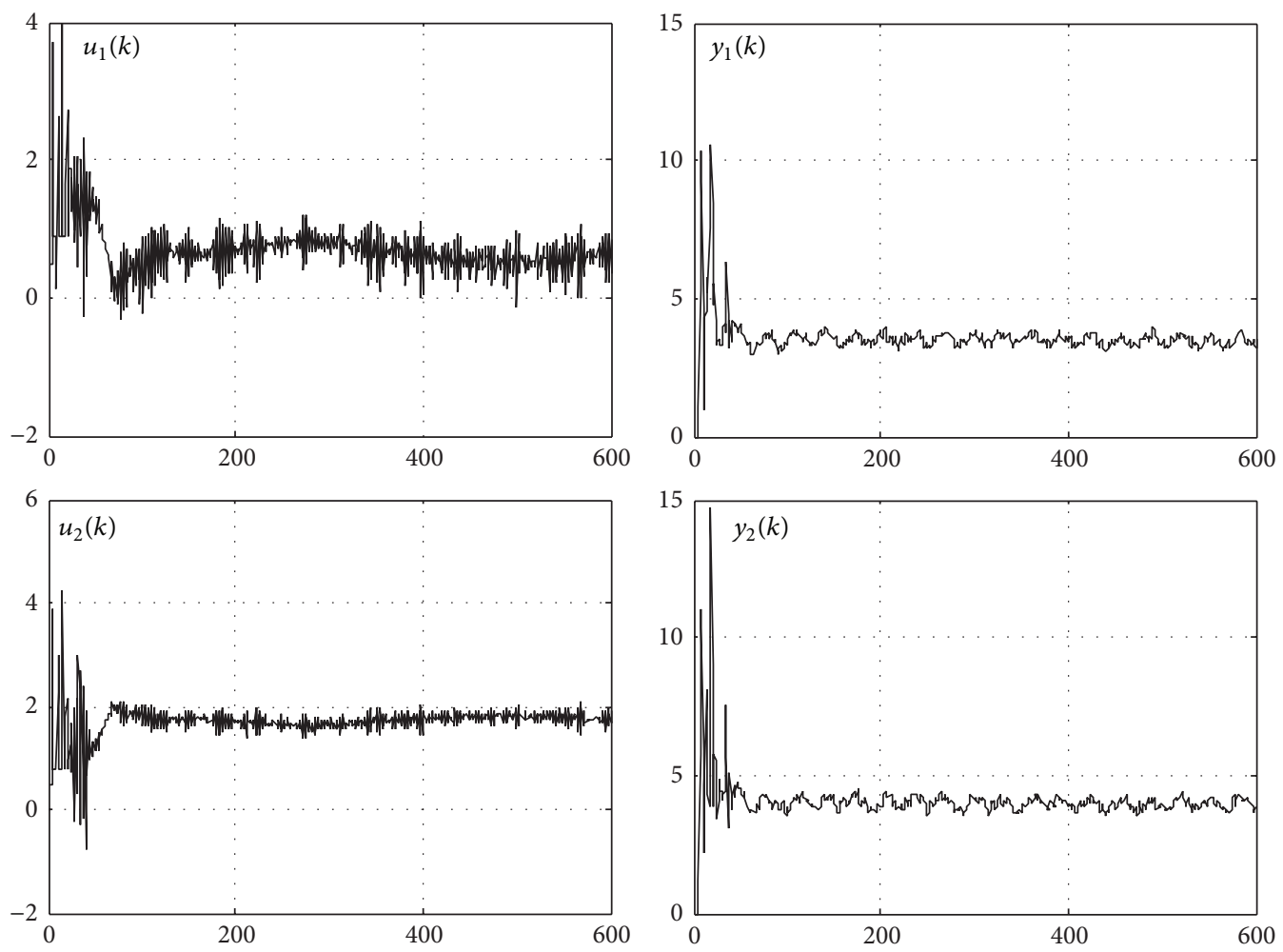

FIGURE 6: Evolution curves of the control laws $u_{1}(k)$ and $u_{2}(k)$ and the outputs $y_{1}(k)$ and $y_{2}(k)$ of the system.
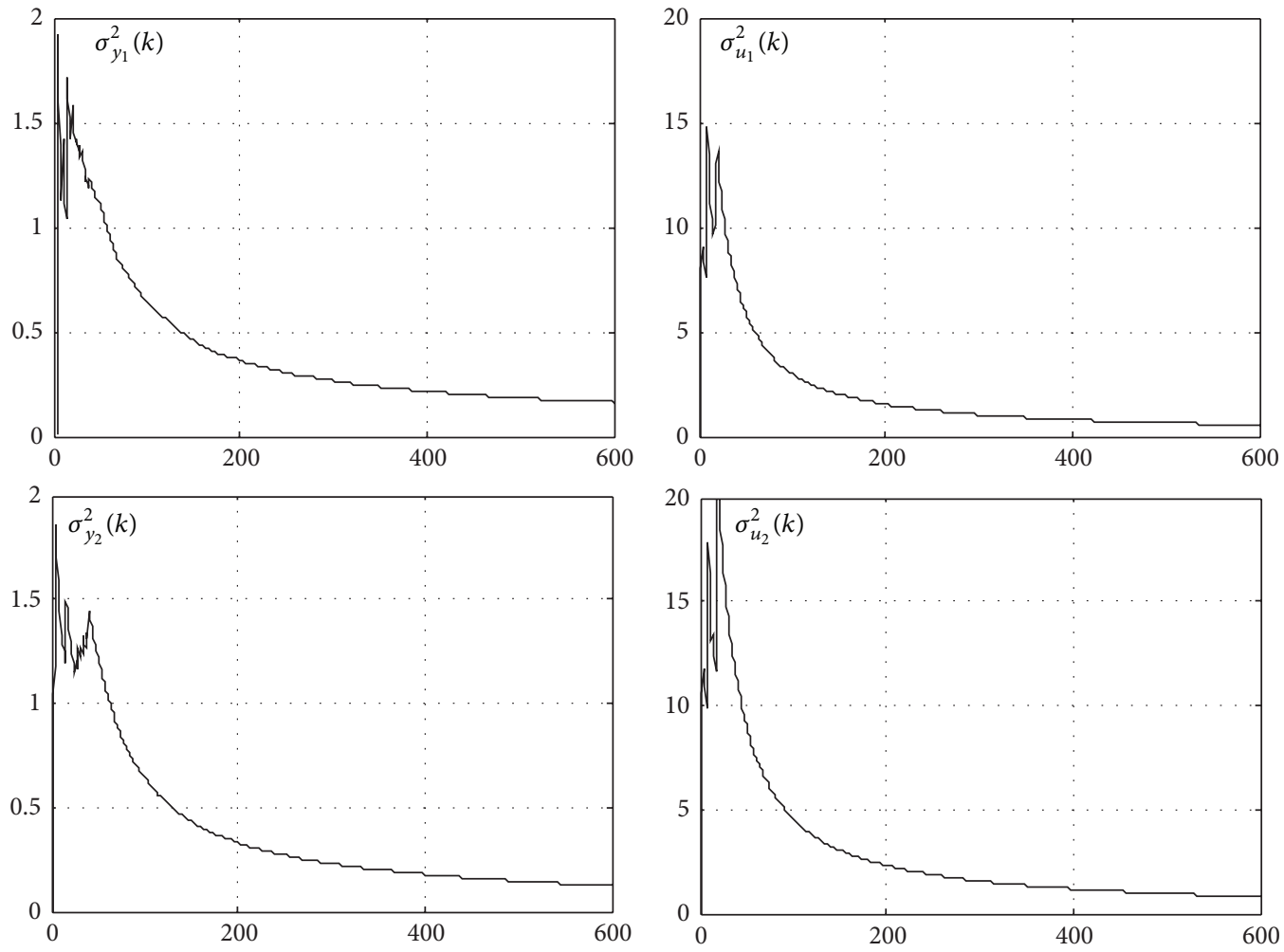

FIGURE 7: Evolution curves of the outputs variances $\sigma_{y_{1}}^{2}(k)$ and $\sigma_{y_{2}}^{2}(k)$ and the control laws variances $\sigma_{u_{1}}^{2}(k)$ and $\sigma_{u_{2}}^{2}(k)$. 
TABLE 2: Statistical average values of the controllers and the outputs signals.

\begin{tabular}{lccc}
\hline $\bar{m}_{u_{1}}$ & $\sigma_{u_{1}}^{2}$ & $\bar{m}_{u_{2}}$ & $\sigma_{u_{2}}^{2}$ \\
0.5942 & 0.5736 & 1.7683 & 0.8629 \\
\hline $\bar{m}_{y_{1}}$ & $\sigma_{y_{1}}^{2}$ & $\bar{m}_{y_{2}}$ & $\sigma_{y_{2}}^{2}$ \\
3.4942 & 0.1822 & 4.0217 & 0.1448 \\
\hline
\end{tabular}

elements, as delineated in Figures 4 and 5, indicate the good quality of the estimate, which is obtained using the RELS algorithm (12). This estimate quality ensures exact quality of control, and it is justified by Figures 6 and 7, which present the evolution curves of the control laws $u_{1}(k)$ and $u_{2}(k)$, the system outputs $y_{1}(k)$ and $y_{2}(k)$, and their variances $\sigma_{y_{1}}^{2}(k)$, $\sigma_{y_{2}}^{2}(k), \sigma_{u_{1}}^{2}(k)$, and $\sigma_{u_{2}}^{2}(k)$.

Table 2 presents the statistical average values $\bar{m}_{u_{i}}$ and $\bar{m}_{y_{i}}$ of the control law $u_{i}(k)$ and the system output $y_{i}(k)$, respectively, and their variances $\sigma_{u_{i}}^{2}$ and $\sigma_{y_{i}}^{2}$ for each interconnected system $S_{i}, i=1,2$. These values were obtained using (46). Note that the evolution curves of the variances $\sigma_{y_{1}}^{2}(k)$ and $\sigma_{y_{2}}^{2}(k)$ converge towards minimum values. Therefore, exact regulation is achieved with the developed self-tuning controller despite the parameters variations of the interconnected nonlinear system, the presence of interactions signals, and disturbances interim on each interconnected system output.

\section{Conclusions}

The self-tuning control problem of large-scale nonlinear discrete-time systems composed of several SISO interconnected nonlinear stochastic subsystems has been proposed in this paper. Each interconnected nonlinear subsystem is described by Hammerstein mathematical model with unknown and time-varying parameters. A recursive algorithm for self-tuning regulation of large-scale interconnected stochastic systems has been developed on the basis of the minimum variance approach with explicit scheme. The presented adaptive control algorithm requires three steps at each control cycle. The general algorithm proposed here is combined with a real-time estimation step and it is carried out to solve the regulation problem for this class of interconnected discrete-time systems by reducing the disturbances acting on the output. The estimation step has been achieved through the adjustable model method and the least-squares techniques. An illustrative simulation example of hydraulic system was treated in order to validate our developed theoretical results.

\section{Competing Interests}

The authors declare that they have no competing interests.

\section{References}

[1] F. Da, "Decentralized sliding mode adaptive controller design based on fuzzy neural networks for interconnected uncertain nonlinear systems," IEEE Transactions on Neural Networks, vol. 11, no. 6, pp. 1471-1480, 2000.
[2] F.-H. Hsiao, C.-W. Chen, Y.-W. Liang, S.-D. Xu, and W.-L. Chiang, "T-S fuzzy controllers for nonlinear interconnected systems with multiple time delays," IEEE Transactions on Circuits and Systems I: Regular Papers, vol. 52, no. 9, pp. 18831893, 2005.

[3] D. D. Šiljak and A. I. Zečević, "Control of large-scale systems: beyond decentralized feedback," Annual Reviews in Control, vol. 29, no. 2, pp. 169-179, 2005.

[4] L. Bakule, "Decentralized control: an overview," Annual Reviews in Control, vol. 32, no. 1, pp. 87-98, 2008.

[5] S. Tong, P. Shi, and H. Al-Madfai, "Robust fuzzy decentralized control for nonlinear large-scale systems with parametric uncertainties," Journal of Intelligent and Fuzzy Systems, vol. 19, no. 2, pp. 85-101, 2008.

[6] A. S. Tlili and N. B. Braiek, "Decentralized observer based guaranteed cost control for nonlinear interconnected systems," International Journal of Control and Automation, vol. 2, no. 2, pp. 29-46, 2009.

[7] S. Kamoun, Description, estimation and optimal self-tuning control of large stochastic systems [Ph.D. thesis], National Engineering School of Sfax, University of Sfax, Sfax, Tunisia, 2009.

[8] S. Kamoun, "Design of optimal self-tuning regulators for largescale stochastic systems," International Journal of Information and Systems Sciences, vol. 6, no. 2, pp. 201-219, 2010.

[9] S. Tong, Y. Li, and X. Jing, "Adaptive fuzzy decentralized dynamics surface control for nonlinear large-scale systems based on high-gain observer," Information Sciences, vol. 235, pp. 287-307, 2013.

[10] S. J. Yoo, "Neural-network-based decentralized fault-tolerant control for a class of nonlinear large-scale systems with unknown time-delayed interaction faults," Journal of the Franklin Institute, vol. 351, no. 3, pp. 1615-1629, 2014.

[11] G. B. Koo, J. B. Park, and Y. H. Joo, "Decentralized fuzzy observer-based output-feedback control for nonlinear largescale systems: an LMI approach," IEEE Transactions on Fuzzy Systems, vol. 22, no. 2, pp. 406-419, 2014.

[12] P. Panagi and M. M. Polycarpou, "Decentralized adaptive approximation based control of a class of large-scale systems," in Proceedings of the IEEE American Control Conference (ACC '08), pp. 4191-4196, Seattle, Wash, USA, June 2008.

[13] P. Panagi and M. M. Polycarpou, "Decentralized fault tolerant control of a class of interconnected nonlinear systems," IEEE Transactions on Automatic Control, vol. 56, no. 1, pp. 178-184, 2011.

[14] H. Yousef, M. Hamdy, and E. El-Madbouly, "Robust adaptive fuzzy semi-decentralized control for a class of large-scale nonlinear systems using input-output linearization concept," International Journal of Robust and Nonlinear Control, vol. 20, no. 1, pp. 27-40, 2010.

[15] S. J. Yoo, N. Hovakimyan, and C. Cao, "Decentralized adaptive control for large-scale nonlinear systems with interconnected unmodelled dynamics," IET Control Theory \& Applications, vol. 4, no. 10, pp. 1972-1988, 2010.

[16] Y.-C. Wang and C.-J. Chien, "Decentralized adaptive fuzzy neural iterative learning control for nonaffine nonlinear interconnected systems," Asian Journal of Control, vol. 13, no. 1, pp. 94-106, 2011.

[17] A. Jajarmi, N. Pariz, S. Effati, and A. V. Kamyad, "Infinite horizon optimal control for nonlinear interconnected largescale dynamical systems with an application to optimal attitude control," Asian Journal of Control, vol. 14, no. 5, pp. 1239-1250, 2012. 
[18] N. Chen, Y. Liu, B. Liu, and W. Gui, "Parametric absolute stabilization for interconnected Lurie time-delay systems with polytopic uncertainty," Asian Journal of Control, vol. 16, no. 1, pp. 225-232, 2014.

[19] M. Elloumi and S. Kamoun, "Design of recursive parametric estimation algorithm for large-scale nonlinear systems described by Wiener mathematical models," International Journal on Sciences and Techniques of Automatic Control \& Computer Engineering, vol. 8, pp. 1950-1965, 2014.

[20] M. Elloumi and S. Kamoun, "Parametric estimation of interconnected nonlinear systems described by input-output mathematical models," International Journal of Automation and Computing, In press.

[21] M. Elloumi and S. Kamoun, "Optimal predictor and implicit self-tuning regulator for a class of Hammerstein large-scale systems," in Proceedings of the 4th International Conference on Systems and Control (ICSC '15), pp. 417-423, IEEE, Sousse, Tunisia, April 2015.

[22] M. Elloumi and S. Kamoun, "An iterative parametric estimation method for Hammerstein large-scale systems: a simulation study of hydraulic process," International Journal of Simulation and Process Modelling, In press.

[23] B.-C. Zheng and G.-H. Yang, "Decentralized sliding mode quantized feedback control for a class of uncertain large-scale systems with dead-zone input," Nonlinear Dynamics, vol. 71, no. 3, pp. 417-427, 2013.

[24] S. Nagai and H. Oya, "Synthesis of decentralized variable gain robust controllers for large-scale interconnected systems with structured uncertainties," Journal of Control Science and Engineering, vol. 2014, Article ID 848465, 10 pages, 2014.

[25] S. Nagai and H. Oya, "Synthesis of decentralized variable gain robust controllers with guaranteed $\mathscr{L}_{2}$ gain performance for a class of uncertain large-scale interconnected systems," Journal of Control Science and Engineering, vol. 2015, Article ID 342867, 11 pages, 2015.

[26] K. J. Åström and B. Wittenmark, "On self tuning regulators," Automatica, vol. 9, no. 2, pp. 185-199, 1973.

[27] Z. Li and R. J. Evans, "Generalised minimum variance control of linear time-varying systems," IEE Proceedings: Control Theory and Applications, vol. 149, no. 1, pp. 111-116, 2002.

[28] J. Zhang, F. Ding, and Y. Shi, "Self-tuning control based on multi-innovation stochastic gradient parameter estimation," Systems \& Control Letters, vol. 58, no. 1, pp. 69-75, 2009.

[29] F. Ding, T. Chen, and Z. Iwai, "Adaptive digital control of Hammerstein nonlinear systems with limited output sampling," SIAM Journal on Control and Optimization, vol. 45, no. 6, pp. 2257-2276, 2007.

[30] F. Ding and T. Chen, "Least squares based self-tuning control of dual-rate systems," International Journal of Adaptive Control and Signal Processing, vol. 18, no. 8, pp. 697-714, 2004.

[31] F. Ding and T. Chen, "A gradient based adaptive control algorithm for dual-rate systems," Asian Journal of Control, vol. 8, no. 4, pp. 314-323, 2006.

[32] F. Ding, Y. Shi, and T. Chen, "Auxiliary model-based leastsquares identification methods for Hammerstein output-error systems," Systems \& Control Letters, vol. 56, no. 5, pp. 373-380, 2007.

[33] F. Ding and T. Chen, "Identification of Hammerstein nonlinear ARMAX systems," Automatica, vol. 41, no. 9, pp. 1479-1489, 2005.
[34] F. Ding, X. Wang, Q. Chen, and Y. Xiao, "Recursive leastsquares parameter estimation for a class of output nonlinear systems based on the model decomposition," Circuits, Systems, and Signal Processing, 16 pages, 2015. 


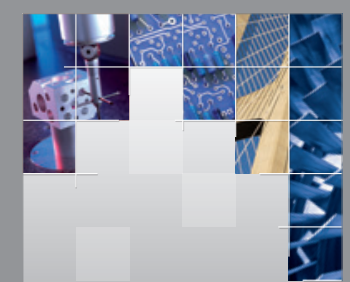

\section{Enfincering}
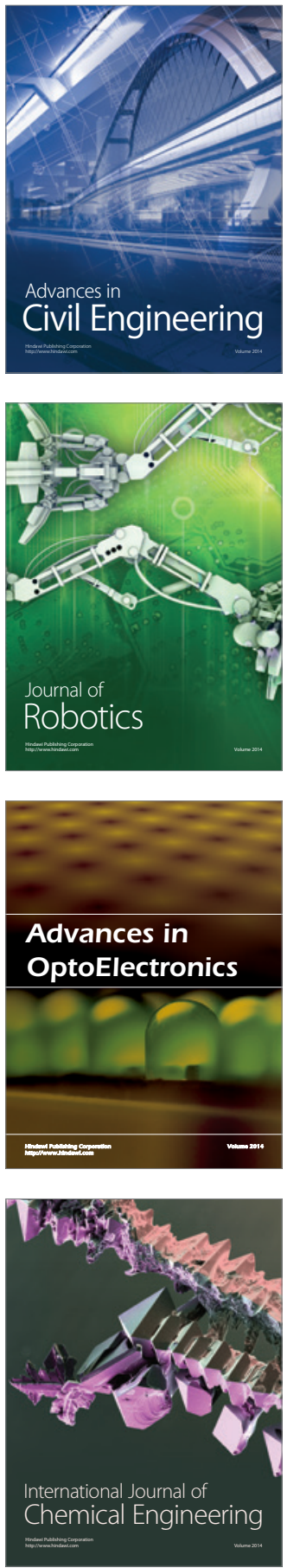

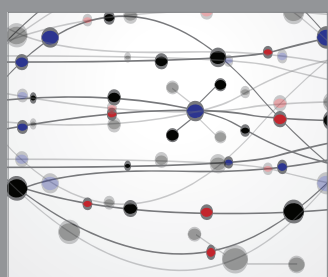

The Scientific World Journal

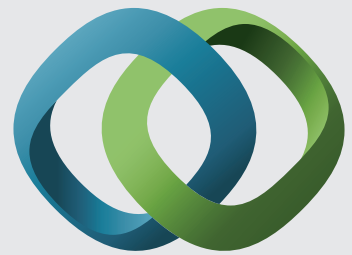

\section{Hindawi}

Submit your manuscripts at

http://www.hindawi.com
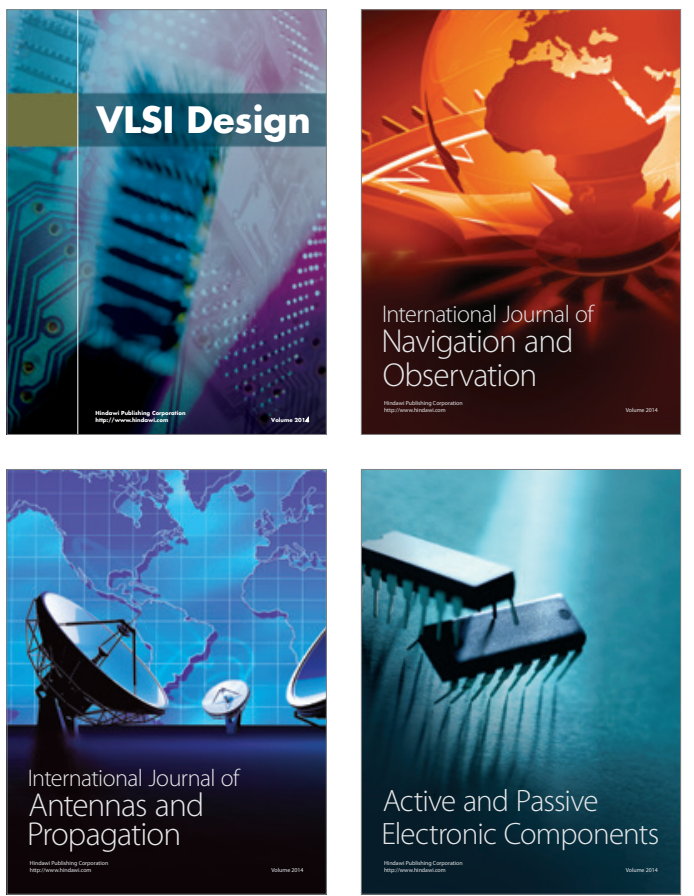
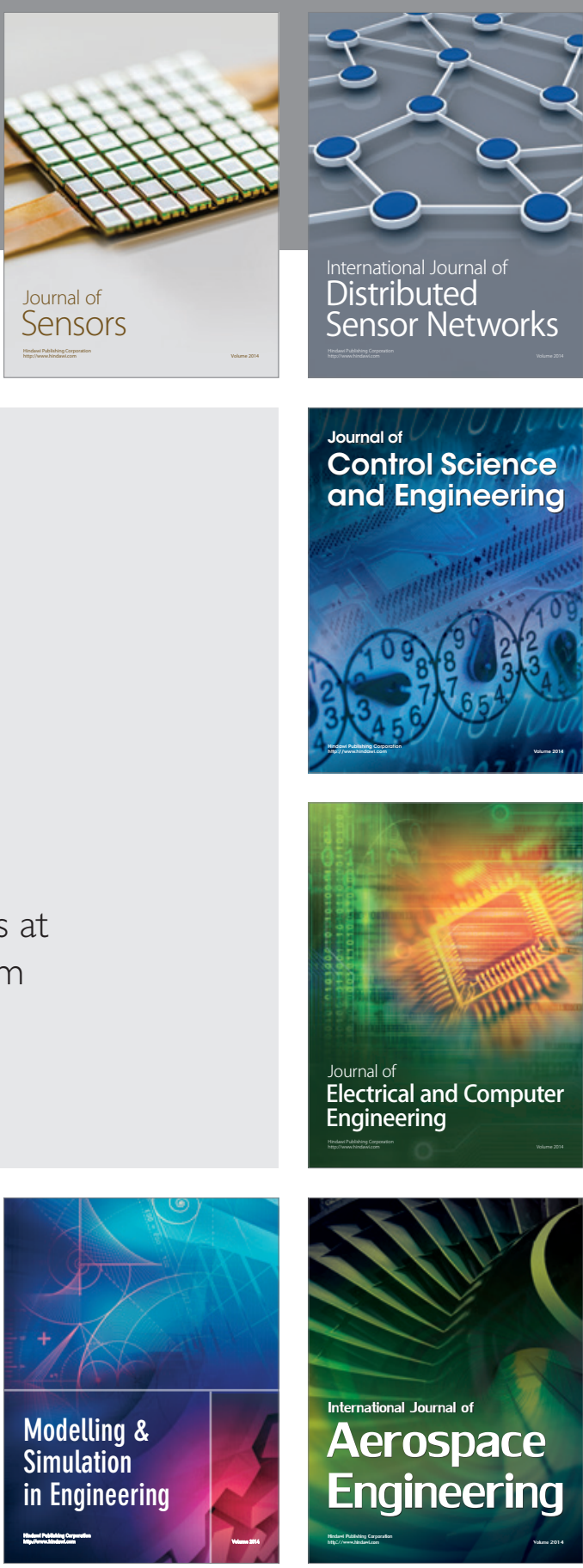

International Journal of

Distributed

Sensor Networks

Journal of

Control Science

and Engineering
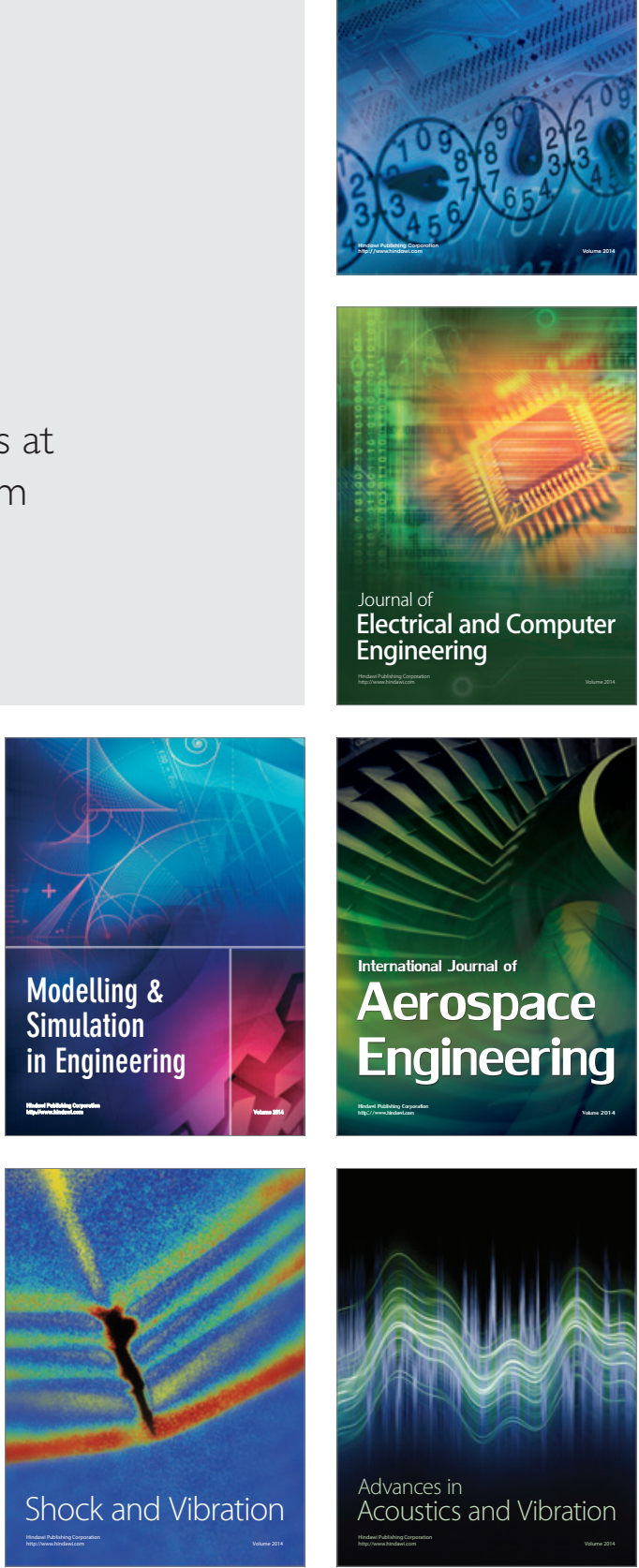